\title{
Sound absorption and reflection from a resonant metasurface: Homogenisation model with experimental validation
}

\author{
Logan Schwan ${ }^{\mathrm{a}, *}$, Olga Umnova ${ }^{\mathrm{b}}$, Claude Boutin ${ }^{\mathrm{c}}$ \\ ${ }^{a}$ LAUM, Université du Maine, UMR CNRS 6613, France \\ ${ }^{\mathrm{b}}$ Acoustics Research Centre, University of Salford, UK \\ ${ }^{\text {c }}$ ENTPE, CeLyA, Université de Lyon, LGCB/LTDS, UMR CNRS 5513, France
}

\section{H I G H L I G H T S}

- Effective surface admittance derived by two-scale asymptotic homogenisation.

- Analytical solution used to design metasurface for sound control at resonance.

- Experimental procedure for metasurface characterisation is developed.

- Total sound absorption at resonance observed in the impedance tube below $400 \mathrm{~Hz}$.

- Near-total sound absorption shown in anechoic chamber with finite size metasurface.

\section{A R T I C L E I N F O}

\section{Article history:}

Received 19 October 2016

Received in revised form 6 February 2017

Accepted 9 February 2017

Available online 12 February 2017

\section{Keywords:}

Acoustic metasurface

Asymptotic homogenisation

Sound absorption

Sound filtering

Enclosure mode control

\begin{abstract}
A B S T R A C T
Efficient manipulation of sound waves by some resonant acoustic metasurface designs has recently been reported in the literature. This paper presents a general theoretical framework for the description of sound wave interaction with the resonant metasurface that is independent of the nature of resonators and the excitation. The equations governing the behaviour of the metasurface are upscaled from the rigorous description of its unit cell using the two scale asymptotic homogenisation. The procedure relies on the existence of the boundary layer confined in the vicinity of the resonators operating in the deep subwavelength regime. The model is capable of describing sound interaction with the array of resonators positioned above or upon the substrate, so that the out of plane direction becomes an additional degree of freedom in the design. It is shown that at the leading order, the behaviour of the resonant surface is described in terms of the effective admittance, whose unconventional properties makes it possible to achieve the total sound absorption at multiple frequencies, broadband absorption, the phase reversal of the reflected wave at resonance and the control of the enclosure modes. The theory is validated by experiments performed in the impedance tube and in the anechoic environment using a surface array of spherical Helmholtz resonators with the extended inner neck. Experimental results confirm the effectiveness and robustness of the resonant surface for control of sound waves.
\end{abstract}

(C) 2017 The Author(s). Published by Elsevier B.V. This is an open access article under the CC BY license (http://creativecommons.org/licenses/by/4.0/).

\footnotetext{
* Corresponding author.

E-mail address: logan.schwan@gmail.com (L. Schwan).
} 


\section{Introduction}

This article is devoted to the theoretical and experimental study of a resonant surface, that is a two-dimensional array of resonators arranged at a surface in a regular lattice. Both resonators and the spacing between them are small compared to the wavelength at the resonance frequency (condition of scale separation). Resonant surfaces are of interest in many domains of physics. They can be used, for instance, to control wavefields through tunable boundary conditions [1-3], or to achieve the perfect absorption of an incident wave [4,5]. In acoustics, examples of resonant surfaces include perforated panels with air cavity behind [6], quarter wavelength tubes folded in a bulky shape [7] or loaded membranes arranged above gas-filled cavity [5]. While the frequency selective absorption is often the targeted function of such surface arrays, other applications are emerging. One of them is to use arrays with graded properties to alter the phase of the reflection coefficient and realise wavefront manipulation [7], somewhat similarly to room diffusers [8]. While resonant surfaces are usually effective in a narrow frequency range close to the resonance, the use of several mistuned resonators per period can broaden the working frequency range [9], but it complicates the design as the period has to remain small compared to the wavelength.

Although several structures have been shown to achieve some remarkable effects in response to some particular incident wavefields (usually plane waves at normal incidence), it seems that a theoretical framework suitable for the description of surface effects in a general case is still lacking. This subject is addressed in this work. Here, a general analytical model based on the method of two-scale asymptotic homogenisation [10-12] is presented which can be applied to any acoustic resonant surface arranged on a rigid backing. No particular assumptions are made about the nature of the resonators or the incident field, except for the condition of scale separation. While the theory of two-scale asymptotic homogenisation is usually applied to find the effective constitutive laws of periodic bulk media, here it is used to derive the effective boundary condition satisfied by the propagating field at the surface array [13-20] by means of a boundary layer analysis. The model provides a systematic analysis of total sound absorption at single or multiple frequencies, broadband absorption, a phase reversal of reflected field at resonance, and control of enclosure mode. In particular, the model addresses the case when the resonators are positioned above the rigid backing, which makes the out-of-plane direction an additional degree of freedom in the design. The theory is validated experimentally using surface arrays of Helmholtz resonators with an extended inner duct designed in $[21,22]$. This design allows for a compact stacking of the resonators at the surface. The experiments are performed in both impedance tube and an anechoic chamber, with the former providing a basic technique for unit cell characterisation and the latter used to characterise absorption by finite-size surfaces.

The article is organised as follows. The two-scale asymptotic homogenisation method is presented in Section 2 and is used for the derivation of an equivalent surface admittance. The unconventional effects accompanying sound reflection from the structured surface are described in Section 3. The approach to the design of the resonant surface is described in Section 4, and the experimental results are presented in Sections 5 and 6.

\section{Resonant surface admittance}

In this section, the propagation of air-borne acoustic waves under ambient conditions is studied in the presence of the resonant surface and in the linear harmonic regime. At equilibrium, the density of air is $\rho_{e}=1.213 \mathrm{~kg} / \mathrm{m}^{3}$, the atmospheric pressure $P_{e}=1.013 \times 10^{5} \mathrm{~Pa}$, the adiabatic constant $\gamma=1.4$, the thermal conductivity $\kappa=0.026 \mathrm{~W} /(\mathrm{Km})$, the heat capacity at constant pressure $c_{p}=1.005 \times 10^{3} \mathrm{~J} /(\mathrm{kg} \mathrm{K})$, the viscosity $\mu=1.85 \times 10^{-5} \mathrm{~Pa} \mathrm{~s}$, and the sound speed $c=\sqrt{\gamma P_{e} / \rho_{e}} \approx 342 \mathrm{~m} / \mathrm{s}$. The study is performed at frequencies $\omega / 2 \pi$ (time convention $e^{-\mathrm{i} \omega t}$ ) close to the natural frequency of the resonators arranged on the surface. Following the model of Boutin \& Roussillon developed for applications in elastodynamics [16], the acoustic behaviour of the resonant surface is described in terms of an effective surface condition derived by means of the two-scale asymptotic homogenisation [10-12]. The approach relies on the scale separation between the long wavelength at resonance and the characteristic lengths of the surface micro-structure, which leads to the existence of a boundary layer confined in the vicinity of the surface array [13-20].

\subsection{Scale separation and boundary layer}

The array under study is the 2-D periodic repetition of the Representative Elementary Volume (REV) $\Omega$ at a plane surface 8, with inward normal vector $\mathbf{n}$ directed at air. The REV $\Omega$ includes $N$ linear acoustic resonators and the rigid backing, see Fig. 1. The nature of the resonators is not specified at this stage. They can be positioned upon or above the rigid backing as well as fully or partially embedded in it. In order to describe this array of three-dimensional REVs by an equivalent surface condition, the homogenisation process must be performed along with a reduction of one space dimension. To do so, the following condition of scale separation is required: the characteristic size $\ell$ of the REV (microscopic scale) has to be much smaller than the reduced wavelength $L=\lambda /(2 \pi)$ (macroscopic scale) of the propagating field in air. This condition is quantified by the scale parameter $\epsilon=\ell / L \ll 1$ which defines the applicability of the model.

The resonators in the REVs experience resonance within the frequency range of scale-separation. To satisfy this condition, a specific design of resonators is required, that leads to a regime of co-dynamics [21] between the microscopic and macroscopic scales. This regime has been described as early as 1985 by Auriault \& Bonnet in elastodynamics [23] (see Ref. [24] for an English version). Since the 2000s, the specific features of this regime have been extensively used to engineer 


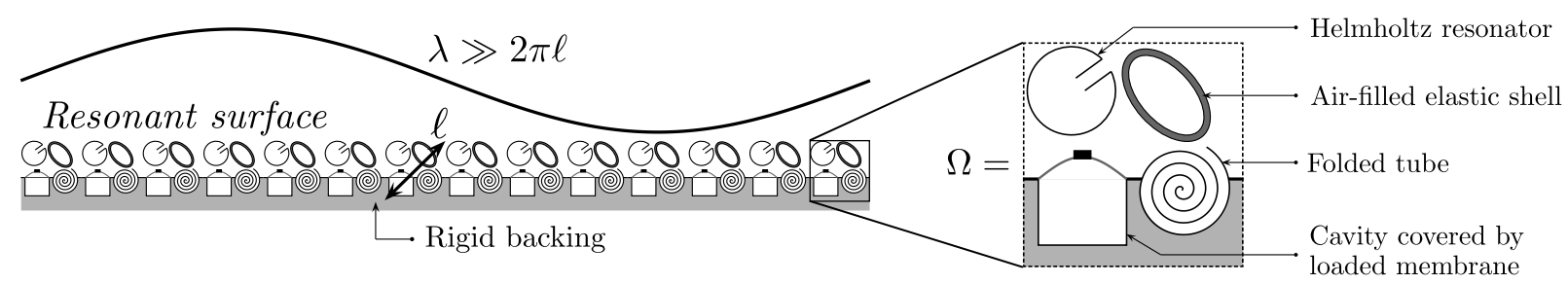

Fig. 1. An example of the resonant surface at a rigid backing. Illustration of the condition of scale separation in 2-D: The size $\ell$ of the REV $\Omega$ is much smaller than the reduced wavelength $\lambda /(2 \pi)$ of the propagating field. Example of resonators in the REV: Helmholtz resonator; air-filled elastic shell; folded tube, and loaded membrane covering cavity.

bulk resonant metamaterials $[25,26]$ and metasurfaces $[15,16,20]$. For structured surfaces, the regime of co-dynamics fundamentally differs from the so-called surface roughness effect $[27,28]$, which is due to surface geometry only (that is, without resonance).

When excited by the long-wavelength propagating field, with the pressure $P$ and the particle velocity $\mathbf{V}$, the resonators of the resonant surface act as mutually-interacting secondary sources that prescribe the particle velocity $\mathbf{v}$ at their boundary. Hence, the velocity distribution at the air/array boundary is locally $\Sigma$-periodic while being modulated at the macroscopic scale. Due to the scale-mismatch with the local periodicity, the long-wavelength propagating field is unable to balance such a velocity distribution on its own. To achieve the scale-transition between the propagating field and the velocity distribution at the boundary, a Boundary Layer (BL) is formed in the vicinity of the surface $[11,14,16,18]$. The BL field, with the pressure $p^{\star}$ and the particle velocity $\mathbf{v}^{\star}$, is superimposed upon the propagating fields $P$ and $\mathbf{V}$ to satisfy the locally-periodic boundary conditions. Under the condition of the scale separation, the boundary layer is confined near the resonant surface, being formed by evanescent waves which fade away at some distance from it. Such diffuse modes [29] inherited from the surface heterogeneity, have been mentioned in some specific cases of surface roughness [27] or membrane arrays [5] in acoustics. In the following, the BL field is shown to be the keystone in the description of the resonant surface in terms of an equivalent boundary condition for the propagating field.

\subsection{Homogenisation scheme}

To describe the resonant surface in terms of an equivalent boundary condition, the method of two-scale asymptotic homogenisation [10-12] is used. This theoretical framework, well-known since the late 1970s, has already been applied to a wide range of physical problems, extended to framed structures [30-32], generalised to micromorphic media [33], and used for media with multiple scales of porosity [34,35]. It is based on the two-scale description of space and asymptotic expansions of the fields. While this method is usually applied to derive the constitutive laws and effective bulk parameters of 3-D periodic media, it can also be adapted for derivation of an equivalent boundary condition at the surface array [13-20]. The present homogenisation scheme combines the usual two-scale asymptotic formulation for periodic continuous media [10-12], with that for the discrete media [30-32] where Taylor expansions of the fields are applied. The homogenisation formulation is described in detail in Appendix A.

This hybrid formulation is justified by the fact that, while the boundary layer is continuous in space, the 2-D periodicity of the array enforces the discretisation of the resonant surface: a countable number of REVs $\Omega_{n}$, where $n$ is an integer, forms the surface array. Similarly, the resonators within the REVs are the discrete entities, which are mutually coupled through the acoustic fields that act at their boundaries. This is evident when defining the local (exact) boundary conditions that have to be satisfied by the total pressure $P+p^{\star}$ and velocity $\mathbf{V}+\mathbf{v}^{\star}$. These conditions are as follows (see Fig. 2 for the notations). In response to the external field $P+p^{\star}$, the resonator $j \in \llbracket 1, N \rrbracket$ of the unit cell $\Omega_{n}$ produces the particle velocity $\mathbf{v}_{n}^{j}=\mathcal{R}^{j}\left(P+p^{\star}\right)$ at its boundary $\Gamma_{n}^{j}$, where the linear operator $\mathcal{R}^{j}$ depends on the inner dynamics of the resonator $j$ (see Section 2.4). In return, the velocity $\mathbf{v}_{n}^{j}$ is balanced by the total velocity field $\mathbf{V}+\mathbf{v}^{\star}$ at the boundary $\Gamma_{n}^{j}$, that is $\left(\mathbf{V}+\mathbf{v}^{\star}\right) \cdot \mathbf{n}_{n}^{j}=\mathbf{v}_{n}^{j} \cdot \mathbf{n}_{n}^{j}$, where the vector $\mathbf{n}_{n}^{j}$ is normal to $\Gamma_{n}^{j}$ and directed at air. The same equations hold at the rigid substrate $\Gamma_{n}^{j=0}$ within $\Omega_{n}$, with $\mathcal{R}^{0} \cdot \mathbf{n}_{n}^{0}=0$.

\subsection{Main results at the leading order}

The most significant results found from two-scale asymptotic homogenisation are summarised here. In the leading order approximation, the boundary layer is shown to be in a quasi-static and quasi-incompressible regime. This is consistent with the fact that, under the condition of scale separation, the BL thickness is much smaller than the wavelength of propagating field. These properties are combined with the local $\Sigma$-periodicity and evanescence of the boundary layer to obtain the following results. First, the BL pressure $p^{\star}$ is shown to be one order of magnitude smaller than that of the propagating field, that is $p^{\star}=\mathcal{O}(\epsilon P)$. For this reason, it can be neglected in the leading order approximation. However, the BL velocity field $\mathbf{v}^{\star}$ cannot be neglected, instead, the BL incompressibility prescribes the following mass conservation law for the macroscopic 


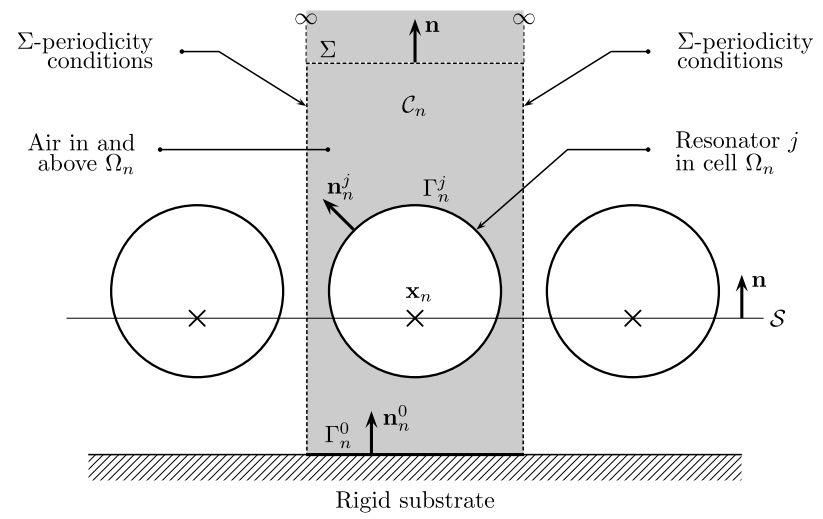

Fig. 2. Schematic at the local scale (three cells are represented) of a resonant surface with a single resonator $j$ in the REV $\Omega_{n}$. The resonator in $\Omega_{n}$ has the boundary $\Gamma_{n}^{j}$ with normal vector $\mathbf{n}_{n}^{j}$. The rigid substrate in $\Omega_{n}$ has the boundary $\Gamma_{n}^{0}$ with normal vector $\mathbf{n}_{n}^{0}$. Representation of the column $\mathcal{C}_{n}$ of air in and above the REV $\Omega_{n}$ where the equilibrium is integrated with $\Sigma$-periodicity conditions applied at its lateral surface, see Appendix A. The plane surface $\&$ and the centroid position $\mathbf{x}_{n}$ of the boundaries $\Gamma_{n}^{j}$ are also shown.

field in the REV $\Omega_{n}$ :

$$
\mathbf{V}\left(\mathbf{x}_{n}\right) \cdot \mathbf{n}=\sum_{j \in \llbracket 1, N \rrbracket} \frac{Q_{n}^{j}}{|\Sigma|} ; \quad Q_{n}^{j}=\int_{\Gamma_{n}^{j}} \mathbf{v}_{n}^{j} \cdot \mathbf{n}_{n}^{j} d \Gamma ;
$$

where $Q_{n}^{j}$ is the acoustic flux produced by the resonator $j$ in the REV $\Omega_{n} ; \mathbf{x}_{n}$ is the centroid of the boundaries $\Gamma_{n}^{j}$ in the REV $\Omega_{n}$; and $|\Sigma|$ is the surface area of the 2-D period $\Sigma$. This means that the propagating field balances the flux produced by all resonators within the REV, per unit area of the 2-D period $\Sigma$. As a result, at the leading order, the exact knowledge of the geometry $\Gamma_{n}^{j}$ or velocity distribution $\mathbf{v}_{n}^{j}$ is not required to formulate the mass conservation law at the boundary. Moreover, any arrangement producing no acoustic flux $\sum_{j} Q_{n}^{j}=0$ (e.g., a distribution of acoustic moments or rigid surface roughness) leads to an effective boundary condition equivalent to that of a rigid surface, that is $\mathbf{V}\left(\mathbf{x}_{n}\right) \cdot \mathbf{n}=0$. Hence, microscopic surface corrugation has no significant effects at the leading order, except for a small phase-shift of the order $\mathcal{O}(\epsilon)$ related to the position of the surface $\&$ relatively to the rigid backing. Note also that Eq. (1) remains valid if the velocity $\mathbf{v}_{n}^{j}$ is prescribed by a periodic array of sources that satisfies the condition of scale separation, e.g., for active metasurface applications [36].

Since the propagating pressure field $P$ prevails at the leading order and is shown to be uniform within the REV $\Omega_{n}$, the velocity $\mathbf{v}_{n}^{j}$ produced by the resonator $j$ in $\Omega_{n}$ results from the pressure $P$ only and reads $\mathbf{v}_{n}^{j}=P\left(\mathbf{x}_{n}\right) \mathcal{R}^{j}(1)$. Using this result, the acoustic flux $Q_{n}^{j}$ produced by the resonator is derived in the form $Q_{n}^{j}=Y^{j} P\left(\mathbf{x}_{n}\right)$, where the admittance $Y^{j}$ is related to the response of resonator $j$ to a unitary pressure field:

$$
Y^{j}=\int_{\Gamma^{j}} \mathcal{R}^{j}(1) \cdot \mathbf{n}^{j} d \Gamma
$$

Note that the admittance $Y^{j}$ is independent on the position $\mathbf{x}_{n}$ because the cells $\Omega_{n}$ are structurally identical. Further, combining Eq. (2) with (1) provides an admittance condition for the propagating fields, $\mathbf{V}^{(0)} \cdot \mathbf{n}=-\Upsilon P$, where the effective admittance reads $\Upsilon\left(\mathbf{x}_{n}\right)=-\sum_{j} Y^{j} /|\Sigma|$. The minus sign is introduced to comply with the convention of defining a surface admittance using the outward normal vector $-\mathbf{n}$. Since under the condition of scale separation the discrete positions $\left\{\mathbf{x}_{n}\right\}$ form a fine mesh on the plane surface $\&$ that includes them, the admittance $\Upsilon$ can be extrapolated to the whole surface [30-32]. As a result, the propagating field is governed by the equations of momentum and mass conservation along with the condition of equivalent admittance:

$$
\mathrm{i} \omega \rho_{e} \mathbf{V}=\operatorname{grad} P ; \quad \mathrm{i} \omega P=\gamma P_{e} \operatorname{div} \mathbf{V} ; \quad \mathbf{V} \cdot \mathbf{n}=-\Upsilon P \quad \text { on } s ; \quad \Upsilon=-\sum_{j \in \llbracket 1, N \|} \frac{Y^{j}}{|\Sigma|} .
$$

The expression for the effective admittance $\Upsilon$ of the array can be seen as the extension of the averaged surface admittance derived for perforated panels with resonators behind [6] to the case when the resonators are located above the rigid surface or only partially embedded in it. As expected from the condition of scale separation $\ell \ll L$, the effective admittance $\Upsilon$ is independent on the thickness of the $\operatorname{REV} \Omega$, contrary to effective fluid layer models [37-39]. Note also that the macroscopic description has been derived exclusively from the description of the system at the microscopic scale and is valid for any propagating field that satisfies the condition of scale separation. The results are accurate up to the precision $\epsilon$. This up-scaling homogenisation method is thus different from other approaches, where the macroscopic effective description is chosen phenomenologically (usually in the same form as that at the microscopic scale) and the effective parameters are 
adjusted to satisfy a rule of micro-macro equivalence (e.g., matching first-order scattering modes [40] or plane wave reflection/transmission coefficients through an array [37-39]). Such a prerequisite-free approach is all the more important for complex media that constitutive equations at the macroscopic scale can depart greatly from those at the microscopic scale, especially when resonances occur. Examples of such atypical up-scaling relations are effective negative mass $[23,24,41]$ or double-gradient models [42] which apply at the macroscopic scale while usual Newton's Law governs the behaviour of the micro-structures.

\subsection{Response function of the resonators}

To describe the response function $\mathcal{R}^{j}$ of the resonator $j$, the dynamic equilibrium of the fields inside the resonator must be considered. The condition of the response linearity and momentum conservation are used, along with Dirichlet and/or Neumann boundary conditions at its surface. Assuming weak inner dissipation, the particle displacement field $\mathbf{u}^{j}$ inside the resonator satisfies the following equation (theorem of virtual work), with $\hat{\mathbf{u}}$ as a test field and $P$ the external pressure field acting at its boundary $\Gamma^{j}$ :

$$
\mathcal{K}^{j}\left(\mathbf{u}^{j}, \hat{\mathbf{u}}\right)-\mathrm{i} \omega \mathcal{L}^{j}\left(\mathbf{u}^{j}, \hat{\mathbf{u}}\right)-\omega^{2} \mathcal{M}^{j}\left(\mathbf{u}^{j}, \hat{\mathbf{u}}\right)=\mathcal{F}^{j}(P, \hat{\mathbf{u}})
$$

where $\mathcal{K}^{j}, \mathcal{L}^{j}$ and $\mathcal{M}^{j}$ are scalar products associated with the stiffness, losses and mass of the resonator $j$ respectively, and $\mathcal{F}^{j}(P, \hat{\mathbf{u}})=-\int_{\Gamma^{j}} P \mathbf{n}^{j} \cdot \hat{\mathbf{u}} d \Gamma$ is the source term. Using normal mode expansion [43], the displacement $\mathbf{u}^{j}$ is given as a series, where the contribution of each mode $m$ takes the form of an eigenvector $\varphi_{m}^{j}$ multiplied by a frequency dependent coefficient:

$$
\mathbf{u}^{j}(\mathbf{x})=\sum_{m} \frac{1}{M_{m}^{j}} \frac{\mathcal{F}^{j}\left(P, \varphi_{m}^{j}\right)}{\left(\omega_{m}^{j}\right)^{2}-\mathrm{i} \zeta_{m}^{j} \omega-\omega^{2}} \boldsymbol{\varphi}_{m}^{j}(\mathbf{x})
$$

where the eigenfrequencies $\omega_{m}^{j}$ satisfy $\mathcal{K}\left(\boldsymbol{\varphi}_{m}^{j}, \hat{\mathbf{u}}\right)=\left(\omega_{m}^{j}\right)^{2} \mathcal{M}\left(\boldsymbol{\varphi}_{m}^{j}, \hat{\mathbf{u}}\right)$, the modal mass is $M_{m}^{j}=\mathcal{M}\left(\boldsymbol{\varphi}_{m}^{j}, \boldsymbol{\varphi}_{m}^{j}\right)$ and the dissipation term is $\zeta_{m}^{j}=\mathcal{L}\left(\boldsymbol{\varphi}_{m}^{j}, \boldsymbol{\varphi}_{m}^{j}\right) / M_{m}^{j} \ll \omega_{m}^{j}$. Since the propagating field is locally uniform on the REV $\Omega_{n}$, that is $P \equiv P\left(\mathbf{x}_{n}\right)$, it can be factorised out of the integral function $\mathcal{F}^{j}$, deriving the displacement $\mathbf{u}_{n}^{j}$ in the resonator $j$ within the $\operatorname{REV} \Omega_{n}$ in the form:

$$
\mathbf{u}_{n}^{j}(\mathbf{x})=-P\left(\mathbf{x}_{n}\right) \sum_{m} \frac{A_{m}^{j} / M_{m}^{j}}{\left(\omega_{m}^{j}\right)^{2}-\mathrm{i} \zeta_{m}^{j} \omega-\omega^{2}} \boldsymbol{\varphi}_{m}^{j}(\mathbf{x})
$$

where $A_{m}^{j}=-\mathcal{F}^{j}\left(1, \varphi_{m}^{j}\right)$ is the modal area. This procedure is illustrated in Appendix B for the case of the quarter-wavelength resonator and the elastic shell with air inside. Note that under a poor scale separation, the field $P$ cannot be assumed uniform on the REV, which leads inevitably to the non-local contributions $[16,44,45]$ in the effective boundary condition (corrector terms in the asymptotic homogenisation).

\subsection{Properties of the effective surface admittance}

The normalised admittance $\rho_{e} c \Upsilon$ of the resonant surface is derived from Eqs. (2), (3) and (6). First, suppose that the REV of the resonant surface contains a single resonator $j$. The frequency range is supposed to be sufficiently close to the eigenfrequency $\omega_{m}^{j}$ for the contributions from all other modes to be negligible in Eq. (6). The normalised admittance takes the form:

$$
\rho_{e} c \Upsilon=\frac{-\mathrm{i} \eta_{m}^{j} \omega}{\omega_{m}^{j 2}-\mathrm{i} \zeta_{m}^{j} \omega-\omega^{2}} ; \quad \eta_{m}^{j}=\frac{\rho_{e} c A_{m}^{j}{ }^{2}}{M_{m}^{j}|\Sigma|},
$$

where $\eta_{m}^{j}$ will be called the admittance parameter (recall that $j$ stands for the resonator and $m$ for the mode). The normalised admittance $\rho_{e} c \Upsilon$ is plotted against frequency in Fig. 3(a). In the low $\left(\omega \ll \omega_{m}^{j}\right)$ and high $\left(\omega \gg \omega_{m}^{j}\right)$ frequency ranges, the admittance $\Upsilon$ is much smaller than that of air, hence the surface acts as an effectively rigid boundary. In contrast, at the resonance $\omega \rightarrow \omega_{m}^{j}$, the normalised admittance is real and equal to $\rho_{e} c \Upsilon=\eta_{m}^{j} / \zeta_{m}^{j}$. This value can be large provided that the resonators are weakly damped, that is $\zeta_{m}^{j} \ll \eta_{m}^{j}$. If several resonators of different types are arranged within the REV, the normalised admittance takes the form:

$$
\rho_{e} c \Upsilon=\sum_{j \in \llbracket 1, N \rrbracket} \sum_{m} \frac{-\mathrm{i} \eta_{m}^{j} \omega}{\left(\omega_{m}^{j}\right)^{2}-\mathrm{i} \zeta_{m}^{j} \omega-\omega^{2}}
$$

where $\eta_{m}^{j}$ has already appeared in Eq. (7). The eigenfrequencies $\omega_{m}^{j}$ can be well separated to produce a sharp admittance contrast at targeted frequencies, or they can be close to each other to broaden the region of high admittance value. These two cases are illustrated in Fig. 3(b) and (c) respectively. Recall however that only the modes satisfying the condition of scale separation are considered here. 

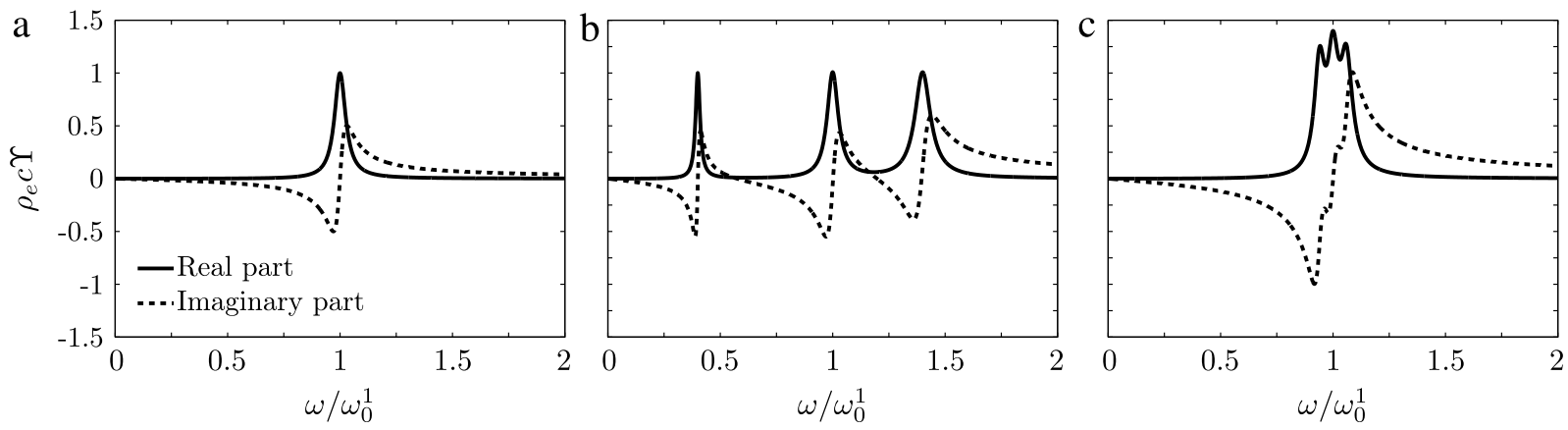

Fig. 3. Real and imaginary parts of the normalised effective admittance against normalised frequency for a metasurface with: (a) one Single Degree Of Freedom (SDOF) resonator per period with the natural frequency $\omega_{0}^{1}$; (b) three SDOF resonators per period, with well separated natural frequencies $\omega_{0}^{2} / \omega_{0}^{1}=0.4$ and $\omega_{0}^{3} / \omega_{0}^{1}=1.4$; and (b) three SDOF resonators per period, with slightly detuned natural frequencies $\omega_{0}^{2} / \omega_{0}^{1}=0.94$ and $\omega_{0}^{3} / \omega_{0}^{1}=1.06$ for broadband effects. Calculations with $\zeta_{0}^{j} / \omega_{0}^{j}=6 \%$ and $\eta_{0}^{j}=\zeta_{0}^{j}$ for each type $j=\{1,2,3\}$ of resonators.

\section{Sound filtering by the resonant surface}

To illustrate the unconventional properties of the admittance $\Upsilon$, plane wave propagation is studied in the presence of the resonant surface. To simplify the analysis, the REV is supposed to include one single degree of freedom resonator with the parameters $\left(\omega_{0}, M_{0}, A_{0}, \zeta_{0}, \varphi_{0}\right)$ and $\eta_{0}=\rho_{e} c A_{0}^{2} /\left(M_{0}|\Sigma|\right)$.

\subsection{Plane wave reflection from the resonant surface}

Reflection of the plane wave $p_{I} e^{\mathrm{i} \omega \mathbf{d}_{I} \cdot \mathbf{x} / c}$ from the admittance surface $s$ is studied, where $p_{I}$ is the complex amplitude and $\mathbf{d}_{I}$ is the unit vector in the direction of propagation. The coordinate system $\left(0, \mathbf{e}_{1}, \mathbf{e}_{2}, \mathbf{n}\right)$ is used, with the origin $O$ on the boundary $\&$. The unit vector $\mathbf{e}_{2} \in \delta$ is in the plane of incidence $\left(\mathbf{d}_{I}, \mathbf{n}\right)$ such that $\mathbf{d}_{I} \cdot \mathbf{e}_{2}>0$. Since the effective surface admittance $\Upsilon$ is uniform, the incident wave gives rise to a reflected wave $p_{R} e^{\mathrm{i} \omega \mathbf{d}_{R} \cdot \mathbf{x} / c}$ which satisfies Descartes' Laws, see Eq. (3). This means that the unit vector $\mathbf{d}_{R}$ belongs to the plane of incidence and has the same horizontal component as the incident field, i.e., $\mathbf{d}_{R} \cdot \mathbf{e}_{2}=\mathbf{d}_{I} \cdot \mathbf{e}_{2}$. The pressure reflection coefficient $R=p_{R} / p_{I}$ takes the usual form:

$$
R=\frac{\cos \theta-\rho_{e} c \Upsilon}{\cos \theta+\rho_{e} c \Upsilon}
$$

where $\theta>0$ is the angle of incidence (counted from the normal $\mathbf{n}$ so that $\mathbf{d}_{R} \cdot \mathbf{n}=-\mathbf{d}_{I} \cdot \mathbf{n}=-\cos \theta$ ). Using Eq. (7), the frequency dependence of the reflection coefficient is derived:

$$
R(\omega)=\frac{\omega_{0}^{2}-\omega^{2}-\mathrm{i} \omega\left(\zeta_{0}-\eta_{0} / \cos \theta\right)}{\omega_{0}^{2}-\omega^{2}-\mathrm{i} \omega\left(\zeta_{0}+\eta_{0} / \cos \theta\right)} .
$$

The reflection coefficient is plotted against frequency in Fig. 4 for different values of the dissipation parameter $\zeta_{0}$ and angles of incidence $\theta$. Far from the resonance $\left(\omega \ll \omega_{0}\right.$ and $\left.\omega \gg \omega_{0}\right)$ it tends to that of the rigid surface $R \rightarrow 1$, consistently with the admittance analysis in Section 2.5. As follows from (10), in the case of no dissipation $\left(\zeta_{0}=0\right)$ the surface is perfectly reflecting for all frequencies and angles of incidence, that is $|R| \equiv 1$. At the resonance frequency, a $\pi$ radian phase shift occurs, independently from the angle of incidence. In that case, the resonant surface acts as an all pass filter with the phase reversal at resonance. In practical applications, however, the dissipation within the resonators cannot be avoided and the introduction of even small dissipation $\left(\zeta_{0} \ll \omega_{0}\right)$ leads to a partial reflection $|R| \leq 1$ and a higher sensitivity to the angle of incidence $\theta$. In particular, around the resonance $\left(\omega \rightarrow \omega_{0}\right)$, the reflection coefficient tends to $R\left(\omega_{0}\right)=\left[\zeta_{0} \cos \theta-\eta_{0}\right] /\left[\zeta_{0} \cos \theta+\eta_{0}\right]$. Hence, the phase-reversal still occurs at resonance provided that $\eta_{0}>\zeta_{0} \cos \theta$. Moreover, if the so-called critical coupling $\cos \theta=\eta_{0} / \zeta_{0} \leq 1$ is achieved [46,47], the reflected wave vanishes at the resonance frequency, $R\left(\omega_{0}\right) \rightarrow 0$. In this case, the resonant surface acts as a band stop filter, i.e., the incident wave of frequency $\omega=\omega_{0}$ is fully absorbed.

\subsection{Memory effect and frequency localisation}

The displacement field $\mathbf{u}_{n}^{j}$ in the resonator $j$ within the REV $\Omega_{n}$, subject to the superposition of the incident and the reflected plane waves $P\left(\mathbf{x}_{n}\right)=(1+R) p_{I} e^{\mathrm{i} \omega \mathbf{d}_{I} \cdot \mathbf{x}_{n} / c}$, is derived using Eqs. (6) and (10):

$$
\mathbf{u}_{n}^{j}(\mathbf{x})=\frac{-2\left(A_{0} / M_{0}\right) p_{I} e^{\mathrm{i} \omega \mathbf{d}_{l} \cdot \mathbf{x}_{n} / c}}{\omega_{0}^{2}-\omega^{2}-\mathrm{i} \omega\left(\zeta_{0}+\eta_{0} / \cos \theta\right)} \boldsymbol{\varphi}_{0}(\mathbf{x}) .
$$



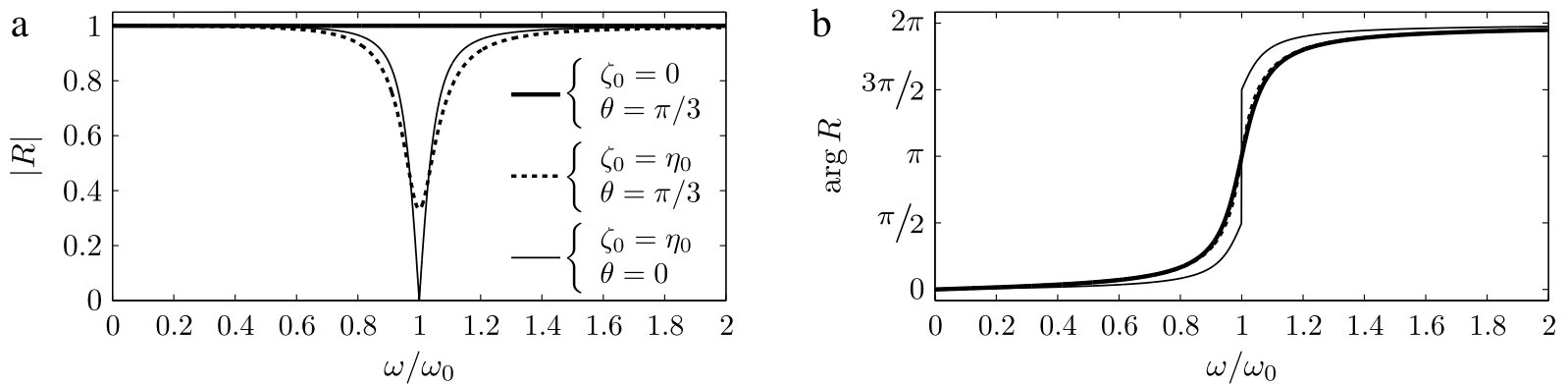

Fig. 4. Plane wave reflection from the resonant surface with resonance frequency $\omega_{0}$. Amplitude (a) and phase (b) of the reflection coefficient $R$ against normalised frequency. Calculations are performed for oblique $(\theta=\pi / 3)$ and normal $(\theta=0)$ incidence assuming weak $\left(\zeta_{0} / \omega_{0}=6 \%\right)$ or no $\left(\zeta_{0}=0\right)$ dissipation and $\eta_{0} / \omega_{0}=6 \%$.

Using convolution, the complex nature of the transfer function $\mathbf{u}_{n}^{j}(\mathbf{x}) / p_{I}$ in the frequency domain can be straightforwardly related to the so-called "memory effect" in the time domain $[11,12,48]$. For instance, if the incident signal is a unitary Dirac delta function, the time dependence of the displacement $\mathbf{u}_{n}^{j}$ at the point $\mathbf{x}$ within the resonator is given by the inverse Fourier transform of Eq. (11). Provided that $\xi=\left(\zeta_{0}+\eta_{0} / \cos \theta\right) /\left(2 \omega_{0}\right)<1$ (weak dissipation), it gives:

$$
\mathbf{u}_{n}^{j}(\mathbf{x}, t)=\frac{-2 A_{0} \varphi_{0}(\mathbf{x})}{M_{0} \widetilde{\omega}_{0}} \sin \left(\widetilde{\omega}_{0}\left(t-t_{n}\right)\right) e^{-\xi \omega_{0}\left(t-t_{n}\right)}
$$

where $t_{n}=\mathbf{d}_{I} \cdot \mathbf{x}_{n} / c$ and $\widetilde{\omega}_{0}=\omega_{0} \sqrt{1-\xi^{2}}$.

The displacement $\mathbf{u}_{n}^{j}$ experiences oscillations with the angular frequency $\widetilde{\omega}_{0} \leq \omega_{0}$ and its amplitude decreases exponentially with the characteristic time $1 /\left(\xi \omega_{0}\right)$. Contrary to the instantaneous response of a rigid surface, the resonant surface thus exhibits a delay in the response: the spectral energy of the incident wave corresponding to the frequency $\widetilde{\omega}_{0}$ is stored within the resonators and is gradually released into the upper half space within the time interval $\mathcal{O}\left(1 /\left(\xi \omega_{0}\right)\right)$.

Even if there is no structural dissipation $\left(\zeta_{0}=0\right)$, the resonators still experience the apparent dissipation $\eta_{0} / \cos \theta$. Due to energy conservation, the energy lost by the resonators is gained by the half-space and the apparent dissipation is therefore attributed to the energy radiation from the resonators. Note that the radiation damping increases with the angle of incidence.

The radiation loss explains the phenomenon of total absorption at resonance presented in Section 3.1: If $\eta_{0} / \cos \theta=$ $\zeta_{0} \ll \omega_{0}$, the energy stored in the resonators is released back into the upper half-space with the same rate $1 / \zeta_{0}$ as it is dissipated within the resonators, thus resulting in no reflected wave. In that case, the spectrum of the reflected field would miss the frequency $\omega_{0}$ while the field inside the resonators would oscillate at the very close frequency $\widetilde{\omega}_{0} \approx \omega_{0}$. Hence, the resonant surface spatially localises the incident energy corresponding to harmonic with frequency $\omega_{0}$ within the deeply subwavelength resonators. The memory effects and localisation of the field could be used to realise acoustic buffers or to be exploited for energy harvesting [5].

\subsection{Control of enclosure mode}

The resonant surface can be used to modify the modal properties of the enclosures, such as rooms, in the low frequency range. Suppose that the enclosure consists of a layer of air bounded between the resonant surface and a rigid wall, see Fig. 5. The standing waves $\psi(x)=A \cos (\omega x / c)+B \sin (\omega x / c)$ in the direction $x$ normal to the boundaries have to satisfy the admittance condition (3) at the resonant surface $x=0$, while $\partial \psi / \partial x=0$ at the rigid wall, $x=H$. These conditions result in the following eigenvectors:

$$
\psi_{q}(x)=\cos \left(\omega_{q} x / c\right)-\mathrm{i} \rho_{e} c \Upsilon\left(\omega_{q}\right) \sin \left(\omega_{q} x / c\right)
$$

where the eigenfrequencies $\omega_{q}$ are the solutions of the following equation, with $q$ being an integer:

$$
\mathrm{i} \sin \left(\omega_{q} H / c\right)-\rho_{e} c \Upsilon\left(\omega_{q}\right) \cos \left(\omega_{q} H / c\right)=0 .
$$

If $\rho_{e} c \Upsilon \rightarrow 0$, the usual modes of the enclosure with rigid walls are recovered, that is $\psi_{q}^{e}(x)=\cos \left(\omega_{q}^{e} x / c\right)$ with $\omega_{q}^{e}=q \pi c / H$. If the natural frequency $\omega_{0}$ of the resonant surface is close to the fundamental frequency $\omega_{1}^{e}=\pi c / H$ of the enclosure with rigid walls, a coupling occurs between the two modes. Using the expression for the normalised admittance $\rho_{e} c \Upsilon$ given in Eq. (7), assuming lossless resonators $\left(\zeta_{0}=0\right)$, and performing Taylor expansions for frequencies $\omega_{q}$ close to both frequencies $\omega_{1}^{e}$ and $\omega_{0}^{j}$, the following leading order approximation is derived for the eigenfrequencies:

$$
\omega_{q}=\bar{\omega} \pm \sqrt{\bar{\omega}^{2}-\omega_{1}^{e} \omega_{0}} ; \quad \bar{\omega}=\frac{\omega_{0}+\omega_{1}^{e}}{2}+\frac{\omega_{1}^{e} \eta_{0}^{j}}{4 \pi} .
$$




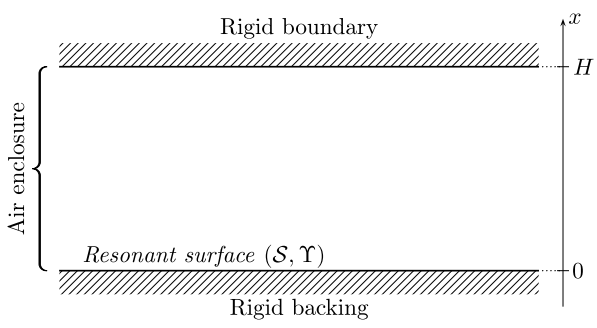

Fig. 5. Enclosure consisting of the layer of air bounded between a rigid wall $(x=H)$ and a resonant surface $(x=0)$.
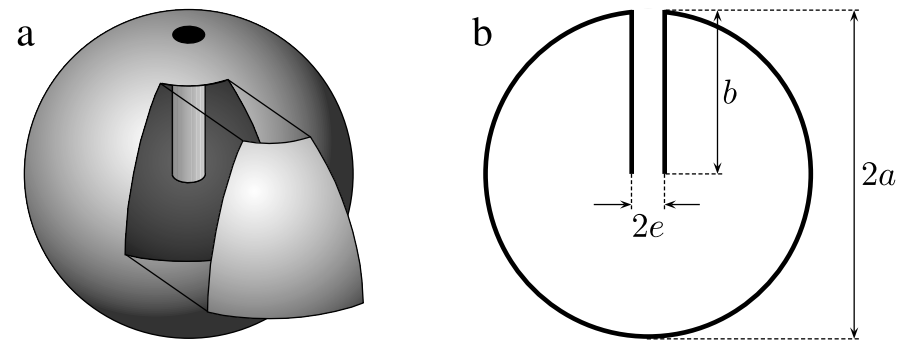

Fig. 6. Spherical Helmholtz resonator: (a) 3-D view; (b) cross section view.

The fundamental mode of the enclosure with rigid walls is split into two modes, the eigenfrequencies of which are either higher or lower than $\bar{\omega}$. Similar results have been reported in other frequency ranges, coupling one or several resonators with cavity modes [49,50] and using the so-called 'tuned mass damper' effect [51]. The main difference here is that the coupling results from the collective behaviour of the large number of resonators experiencing deep subwavelength resonance.

\section{Design and characterisation of the resonant surface}

In the experimental part of this work, impedance tube and anechoic chamber measurements are performed on the resonant surface made of multiple Helmholtz resonators. In this section, an approach to the resonator design and characterisation is described.

\subsection{Design}

The resonant surface consists of the spherical Helmholtz resonators arranged periodically upon the rigid backing. The resonator's spherical cavity of radius $a$ is connected to a straight circular duct of length $b$, radius $e$ and cross-section area $A=\pi e^{2}$, see Fig. 6 . For practicality, the duct is positioned inside the cavity [21]. Since dissipation has been identified as a key mechanism defining reflection properties of the surface (Section 3.1), a detailed description of the losses within the resonators is given.

In the first approximation, valid when the scale separation is sharp [21], air in the cavity is assumed quasi-static, while that in the duct is incompressible. This results in the uniform pressure field $\widetilde{p}$ in the cavity, and the pistonic motion of air in the duct with the displacement $\grave{u}$, positive in the direction away from the resonator. Also, the viscous losses in the cavity and the thermal losses in the duct can be neglected, since grad $\widetilde{p}=\mathbf{0}$ and divù $=0$.

When the air is pulsed out from the resonator through the duct, the decrease of pressure in the cavity is $\tilde{p}=$ $-\beta \gamma P_{e} A\langle\grave{u}\rangle / V$, where $V=4 \pi a^{3} / 3-A b$ is the cavity volume, $\beta \gamma P_{e}$ is the effective bulk modulus of air [52], and $\langle\grave{u}\rangle=$ $A^{-1} \int_{A} \grave{u} d S$ is the particle displacement averaged over the cross-section of the duct. That latter satisfies the equation of momentum conservation $-\omega^{2} \tau \rho_{e}\langle\grave{u}\rangle=(\widetilde{p}-P) / b$ where $\tau$ is the dynamic tortuosity [52], and $P$ is the pressure acting on the duct aperture from the outside (that is, the propagating field). Combining the two equations, the admittance $Y^{j}=-i \omega A\langle\grave{u}\rangle / P$ of the resonator is derived. If the REV of the surface contains a single Helmholtz resonator, the effective admittance $\Upsilon=-Y^{j} /|\Sigma|$ normalised by that of air is:

$$
\rho_{e} c \Upsilon=\frac{-\mathrm{i} \omega \hat{\eta} / \tau}{\hat{\omega}_{0}^{2} \beta / \tau-\omega^{2}}
$$

where $M=\rho_{e} A b$ is the mass of air in the duct, $\hat{\omega}_{0}=c \sqrt{A /(b V)}$ is the natural frequency and $\hat{\eta}=\rho_{e} c A^{2} /(M|\Sigma|)$ the admittance parameter for lossless resonators. The duct tortuosity $\tau$ and the normalised bulk modulus $\beta$ of air in the spherical cavity (neglecting the presence of the duct), are given by $\tau=1 /(1-F)$ and $\beta=1 /[1+(\gamma-1) G]$, where the functions $F$ 
and $G$ depend on the viscous and thermal skin depths $\delta_{v}=\sqrt{2 \mu /\left(\omega \rho_{e}\right)}$ and $\delta_{T}=\sqrt{2 \kappa /\left(\omega \rho_{e} c_{p}\right)}$ in the form:

$$
F=\frac{2 \delta_{v}}{e \sqrt{2 \mathrm{i}}} \frac{J_{1}\left(\frac{e \sqrt{2 \mathrm{i}}}{\delta_{v}}\right)}{J_{0}\left(\frac{e \sqrt{2 \mathrm{i}}}{\delta_{v}}\right)} ; \quad G=\frac{3 \delta_{T}}{a \sqrt{2 \mathrm{i}}} \frac{j_{1}\left(\frac{a \sqrt{2 \mathrm{i}}}{\delta_{T}}\right)}{j_{0}\left(\frac{a \sqrt{2 \mathrm{i}}}{\delta_{T}}\right)} .
$$

Here, $J_{n}$ and $j_{n}$ are cylindrical and spherical Bessel functions of the first kind and order $n$. Around the resonance and for viscous and thermal skin depths $\delta_{v}$ and $\delta_{T}$ much smaller than the duct and cavity radii $e$ and $a$ (weak dissipation in the resonators), the normalised effective admittance in Eq. (16) can be approximated by that in Eq. (7), that is $\rho_{e} c \Upsilon \rightarrow-i \eta \omega /\left[\omega_{0}^{2}-\mathrm{i} \zeta \omega-\omega^{2}\right]$, with the resonance frequency $\omega_{0}$, the $-3 \mathrm{~dB}$ frequency bandwidth of the resonance peak $\zeta$, and the admittance parameter $\eta$ given by:

$$
\frac{\omega_{0}}{\hat{\omega}_{0}} \approx 1-v ; \quad \frac{\zeta}{\omega_{0}} \approx v ; \quad \frac{\eta}{\hat{\eta}} \approx 1-\frac{\delta_{v}^{0}}{e}
$$

where $v=\delta_{v}^{0} / e+3(\gamma-1) \delta_{T}^{0} /(2 a) \ll 1$ and $\delta_{v}^{0}$ and $\delta_{T}^{0}$ are viscous and thermal skin depths corresponding to frequency $\hat{\omega}_{0}$. Design limitations can now be discussed for the normalised admittance to be significant at the resonance frequency under the condition of scale separation, that is $\left|\rho_{e} c \Upsilon\left(\omega_{0}\right)\right|=\eta / \zeta \geq \mathcal{O}(1)$ with $\epsilon_{0}=\ell \omega_{0} / c \ll 1$. Neglecting viscous and thermal effects, the scale parameter at the resonance, $\hat{\epsilon}_{0}=\ell \hat{\omega}_{0} / c$, and the admittance parameter $\hat{\eta}$ satisfy the following conditions:

$$
\hat{\epsilon}_{0}=\sqrt{\frac{\ell^{2} A}{b V}} ; \quad \frac{\hat{\eta}}{\hat{\omega}_{0}}=\frac{A}{|\Sigma|} \sqrt{\frac{V}{b A}} .
$$

Since the volume $V$ of the cavity is of the order of the REV's volume, that is $V=\mathcal{O}(|\Sigma| \ell)$, Eq. (19) leads to the following estimates: $A=\mathcal{O}\left(\hat{\epsilon}_{0}^{2}|\Sigma| b / \ell\right)$ and $\hat{\eta} / \hat{\omega}_{0}=\mathcal{O}\left(\hat{\epsilon}_{0}\right)$. Using these estimates and Eq. (18), the condition $\left|\rho_{e} c \Upsilon\left(\omega_{0}\right)\right|=\eta / \zeta \geq \mathcal{O}(1)$ leads to the following constraint for viscous and thermal losses: $v \leq \mathcal{O}\left(\hat{\epsilon}_{0}\right)$. To achieve that, the duct radius has to be one order larger than the viscous skin depth, that is $e \geq \mathcal{O}\left(\hat{\epsilon}_{0}^{-1} \delta_{v}\left(\hat{\omega}_{0}\right)\right)$.

If the resonance frequency is around $300 \mathrm{~Hz}$, the viscous and thermal skin depths are $\delta_{v}\left(\hat{\omega}_{0}\right) \approx 0.13 \mathrm{~mm}$ and $\delta_{\tau}\left(\hat{\omega}_{0}\right) \approx$ $0.15 \mathrm{~mm}$. Assuming a scale parameter $\hat{\epsilon}_{0}=\mathcal{O}\left(10^{-1}\right)$, the duct radius would need to be $e \geq \mathcal{O}(1 \mathrm{~mm})$, while the REV size is $\ell=\hat{\epsilon}_{0} c / \hat{\omega}_{0}=\mathcal{O}(1 \mathrm{~cm})$, that is $e=\mathcal{O}\left(\epsilon_{0} \ell\right)$. To satisfy the condition of scale separation $\pi e^{2}=\mathcal{O}\left(\epsilon_{0}^{2} \ell^{2}(b / \ell)\right)$ the duct length has to be of the order of the REV size $b=\mathcal{O}(\ell)=\mathcal{O}\left(\epsilon_{0}^{-1} e\right)$. This means, that the so-called extended neck [53] has to be designed. Therefore, the slotted cylinders [54] and split hollow spheres [55] are not suitable resonators to design the surfaces with a sharp scale separation at resonance. Similar constraints have been found to achieve the regime of codynamics between the subwavelength Helmholtz resonators and a porous matrix [21]. Finally, since $a=\mathcal{O}(\ell)=\mathcal{O}\left(\epsilon_{0}^{-1} e\right)$ and viscous and thermal skin depths are of the same order, then $\delta_{T} / a=\mathcal{O}\left(\epsilon \delta_{v} / e\right)$, that is viscous losses in the duct are dominant over the thermal losses in the cavity.

\subsection{Experimental characterisation of unit cells}

Similarly to Ref. [22], the Helmholtz resonator is constructed by drilling a hole in a $4 \mathrm{~cm}$-diameter table tennis ball and gluing a piece of sipping straw to it, so that it fits inside the ball as shown in Fig. 6. Such resonators have been characterised using a $B \& K$ type 4206 impedance tube with a circular cross-section (diameter $D_{T}=10 \mathrm{~cm}$ ) and a variable length $30 \mathrm{~cm} \leq H \leq 50 \mathrm{~cm}$. It is shown in Appendix A.4 that, in the leading order approximation, the propagating field in the tube satisfies Eqs. (3), with the period area $|\Sigma|$ replaced by that of the tube cross-section $\left|\Sigma_{T}\right|$, independently of its exact geometry. As shown previously for square tubes [56], impedance tube measurements can be used to emulate the reflection of plane waves at normal incidence from a resonant surface having a period area $\left|\Sigma_{T}\right|$. This provides a straightforward method to retrieve the effective admittance $\Upsilon$ of the resonant surface.

With a single resonator in the tube, the normalised admittance $\rho_{e} c \Upsilon$ has one resonance peak, see Fig. 3(a). The resonator's parameters, that is the resonance frequency $\omega_{0} /(2 \pi)$ and the $-3 \mathrm{~dB}$ frequency bandwidth $\zeta /(2 \pi)$ of the resonance peak, are easily deduced. Experimental results are presented in Fig. 7 for resonators with the duct diameters $2 e=\{4,5,6,7\} \mathrm{mm}$ and the duct lengths $b=\{5,10,15,20,25,30\} \mathrm{mm}$. As expected, the wider the duct, the higher the resonance frequency and the weaker the dissipation. The data is in a good agreement with the model predictions when ducts are sufficiently long to provide a resonance frequency so that $2 a \omega_{0} / c<0.3$. For shorter ducts, when the scale separation is not sharp, the model predictions overestimate the natural frequencies $\left(\approx 13 \%\right.$ error for $b=10 \mathrm{~cm}$ ) and underestimate the loss factor $\zeta / \omega_{0}$ (by an amount of $\approx 2.4 \%$ for $b=10 \mathrm{~cm}$ ). This can be attributed to phenomena neglected in the leading order approximation, such as, for example, end corrections at the duct extremities. Experimentally, for a given duct diameter the loss factor $\zeta / \omega_{0}$ is found to be quasi-uniform over the whole range of duct lengths.

The results confirm that a wide range of natural frequencies (180-616 Hz here) and low levels of dissipation $\left(\zeta / \omega_{0} \leq\right.$ 0.11 ) can be achieved with the adopted design of Helmholtz resonators, by simply varying the duct length and its diameter. For natural frequencies below $400 \mathrm{~Hz}$, the wavelength $\lambda$ at the resonance is more than 21 times larger than the diameter of the resonator, which validates the design for deep subwavelength applications. 

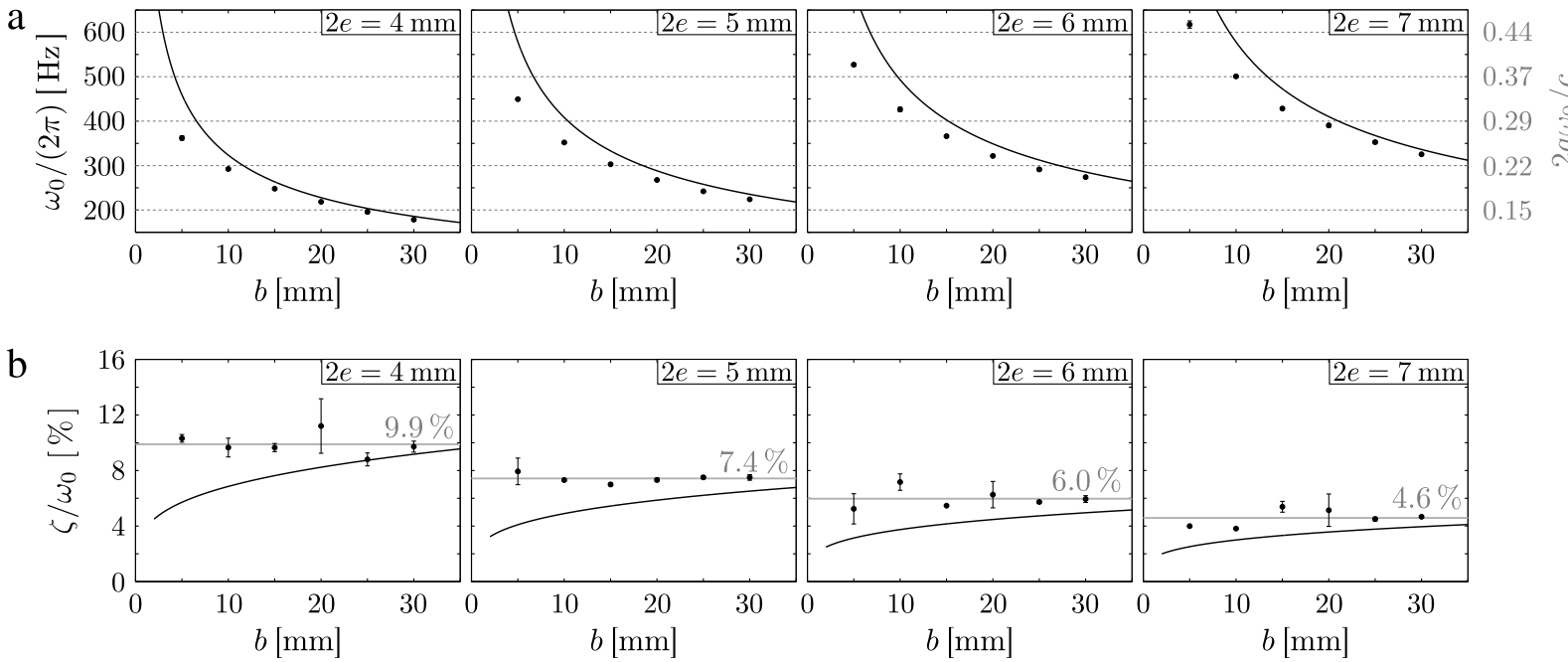

Fig. 7. Modal parameters of spherical Helmholtz resonators with extended neck for 4 duct diameters $2 e=\{4,5,6,7\} \mathrm{mm}$ and six duct length $b=\{5,10,15,20,25,30\} \mathrm{mm}$ : (a) resonance frequency $\omega_{0} /(2 \pi)$; (b) loss factor $\zeta / \omega_{0}$ and mean value over the range of duct lengths $b$ (grey line). For each geometry, three specimen have been tested: the mean value (dots) and the standard deviation (error-bar) of the parameters are presented here. Theoretical predictions (plain line) are shown for comparison. In Figure (a), the diameter $2 a$ of the resonator normalised by the reduced wavelength $c / \omega_{0}$ at the resonance frequency is shown (grey dashed lines).
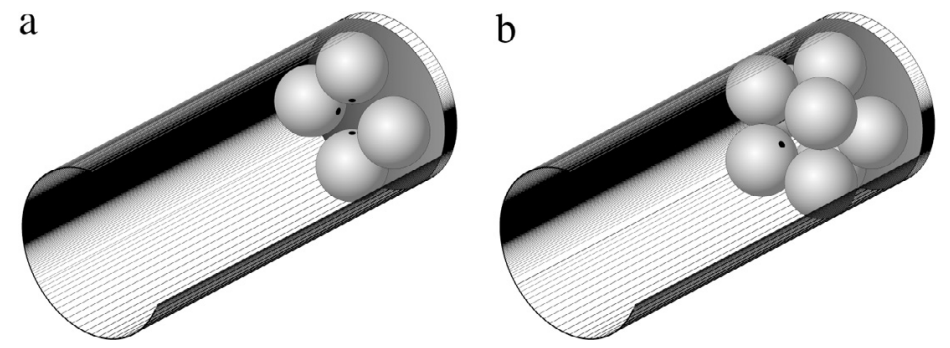

Fig. 8. Compact arrangements of spherical Helmholtz resonators at the rigid end of the impedance tube: (a) single layer; (b) double layer.

\section{Impedance tube measurements}

In this section, impedance tube measurements of sound absorption and enclosure mode control using the resonant surface designed in Section 4 are described.

\subsection{Experimental configurations}

The configurations tested are presented in Table 1. Along with the Helmholtz resonators, rigid spheres of the same size have been added in the tube in order to achieve configurations with either 4 or 8 scatterers in the tube. Four scatterers are arranged symmetrically in a single layer at the rigid end of the tube, see Fig. 8(a). Eight scatterers are arranged in two layers, with positions of those in the upper layer shifted by the angle $\pi / 4$ compared to the lower layer, to achieve a higher degree of compaction, see Fig. $8(\mathrm{~b})$. The centroid of the surface structures is at the distance $x_{s}^{\mathrm{SL}} \approx 1.4 \mathrm{~cm}$ for the single layer, and $x_{s}^{\mathrm{DL}} \approx 3.1 \mathrm{~cm}$ for the double layer, see Appendix A. It has been checked experimentally that the relative positions of the scatterers (resonators and rigid spheres) and the orientations of the resonators' duct apertures have negligible influence on the results. For the measurements described here, the duct apertures are directed towards the main axis of the tube.

Even with a double layer configuration, the thickness of the arrangement is about $7.25 \mathrm{~cm}$ which is less than the tube diameter $D_{T}=10 \mathrm{~cm}$. Hence, the characteristic size of the REV is $\ell_{T}=D_{T}$ and the scale parameter $\epsilon_{T}=\ell_{T} \omega / c$ remains below 1 for frequencies below $c /\left(2 \pi \ell_{T}\right)=544.3 \mathrm{~Hz}$. In this section, the data is compared with the admittance model predictions using either Eq. (16) or Eq. (8) with values of parameters $\omega_{0}$ and $\zeta$ measured in Section 4.2.

\subsection{Sound absorption}

The configurations $A$ to $D$ are designed to achieve the critical coupling $\eta \approx \zeta$ for the duct diameters $2 e=\{4,5,6,7\} \mathrm{mm}$. Experimental results are shown in Fig. 9(a). As expected a nearly total absorption of sound is achieved at the resonance, 
Table 1

Resonant surface configurations (Conf.) tested in the impedance tube: values of the duct diameter $2 e$, duct length $b$, number of resonators $N$, and impedance tube length $H$. Theoretical estimates for the resonance frequency $\omega_{0} /(2 \pi)$, and scale parameter at the resonance $\epsilon_{0}=$ $D_{T} \omega_{0} / c$ are given.

\begin{tabular}{lllllll}
\hline Conf. & $2 e(\mathrm{~mm})$ & $b(\mathrm{~mm})$ & $N$ & $H(\mathrm{~cm})$ & $\omega_{0} /(2 \pi)(\mathrm{Hz})$ & $\epsilon_{0}$ \\
\hline A & 4 & 25 & 7 & 38 & 203 & 0.37 \\
B & 5 & 15 & 4 & 38 & 333 & 0.61 \\
C & 6 & 20 & 3 & 38 & 349 & 0.61 \\
D & 7 & 23 & 2 & 38 & 383 & 0.7 \\
E & 7 & 23 & 4 & 38 & 383 & 0.7 \\
F & 7 & 23 & 4 & 48 & 383 & 0.7 \\
\hline
\end{tabular}
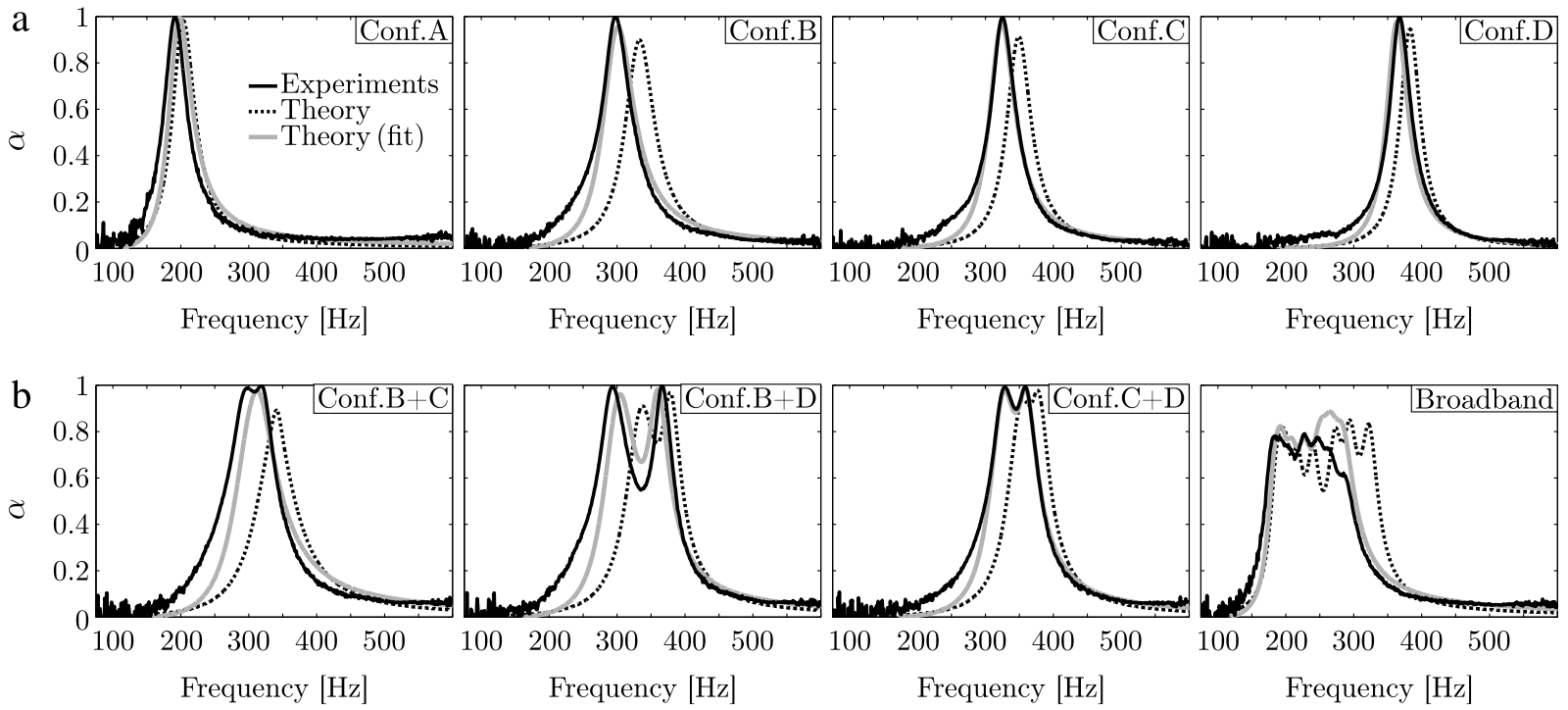

Fig. 9. Absorption coefficient for: (a) configurations $A$ to $D$ providing a nearly total sound absorption at the resonance frequencies; and (b) combinations of detuned resonators. Comparison of impedance tube measurements with the model using analytical results (dashed line) and resonators' parameters $\omega_{0}$ and $\zeta$ deduced from characterisation tests (grey line).

with absorption coefficients equal to $\alpha_{\exp }\left(\omega_{0}\right)=\{0.9973,0.9999,0.9957,0.9977\}$ (theoretical predictions are $\alpha\left(\omega_{0}\right) \approx$ $\{0.98,0.899,0.914,0.947\})$ for the configuration $A$ to $D$ respectively. The nearly total absorption of sound can be achieved at several frequencies by combining resonators with different natural frequencies. This is demonstrated in Fig. 9(b), where the absorption coefficients are presented for double layer configurations of resonators with different resonance frequencies, that is $B+C, B+D$ and $C+D$. According to the theoretical results, described in Section 2.5, when the two resonance frequencies are close to each other, a broader absorption peak is observed, see configurations $B+C$ or $C+D$. Broadband effects are further investigated by combining eight resonators having a $4 \mathrm{~mm}$-diameter duct and the duct lengths $b=$ $\{10,12,14,18,22,26,28,30\} \mathrm{mm}$ in a double layer configuration. The natural frequencies of these resonators are ranging from 179 to $290 \mathrm{~Hz}$. The absorption coefficient of this configuration is shown in Fig. 9(b), with values higher than 0.6 in the frequency range [173-288] Hz. Despite a relatively poor scale separation (limited by the impedance tube diameter), the theory is in a good agreement with the experiments, particularly when the measured values of the resonator parameters $\omega_{0}$ and $\zeta$ are used.

\subsection{Phase reversal upon reflection}

Configuration $E$ is designed so that a $\pi$ radian phase shift in the reflection coefficient occurs at the resonance. For normally incident plane wave, that happens if $\eta / \zeta>1$, see Section 3.1. The amplitude and phase of the reflection coefficient are shown as functions of frequency in Fig. 10. The phase reversal is observed in the experiments and the model predictions agree well with the data. Note that the reflection coefficient amplitude is relatively low at resonance, $\left|R\left(\omega_{0}\right)\right| \approx 0.36$, despite the weak dissipation (loss factor $\zeta / \omega_{0} \approx 4.6 \%$ ). This means that reflection from a resonant surface is very sensitive to damping, which cannot be neglected for practical applications, such as wavefront manipulation [7]. 

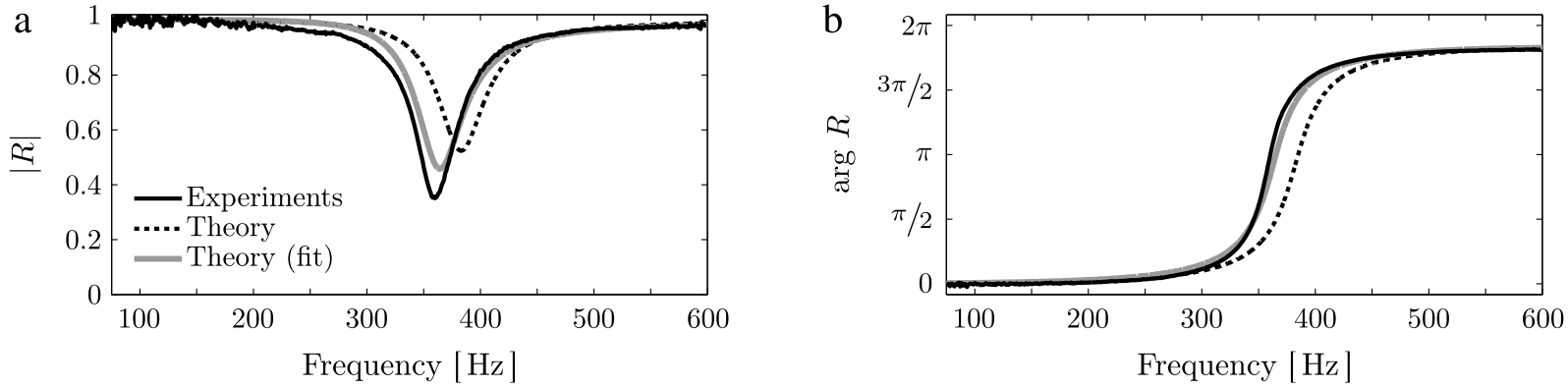

Fig. 10. Reflection coefficient $R$ against frequency for configuration $E$ : (a) amplitude; and (b) phase. Comparison of experimental results with the admittance model predictions using analytical results (dashed line) and modal properties deduced from characterisation tests (grey line).
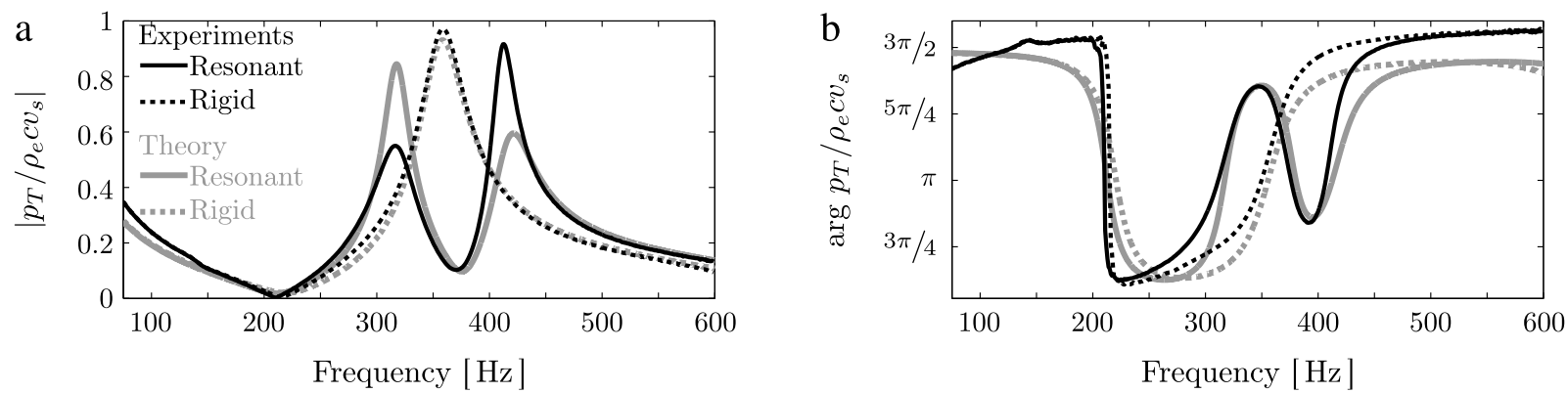

Fig. 11. Normalised pressure $p_{T}$ in the impedance tube at $x=39 \mathrm{~cm}$ for configuration $F$ : (a) amplitude; and (b) phase against frequency. Comparison of experimental results with admittance model predictions for the tube with the resonant surface and the rigid termination. Modal properties fitted on characterisation tests have been used in the model.

\subsection{Control of enclosure modes}

The resonant surface in configuration $F$ is identical to that in configuration $E$, but the tube length $H$ has been chosen so that the resonance frequency $c /(2 \mathrm{H})$ of the tube with the rigid termination is close to that of the resonant surface. That allows to control the enclosure mode by the resonant surface. The pressure field $p_{T}(x)$ in the tube satisfies the admittance condition (3) at $x_{s}=x_{\S}^{\mathrm{SL}}$ and the particle velocity $v_{s}$ is prescribed by the speaker at $x=H$. For better accuracy, dissipation in the tube is accounted for by considering a complex wavenumber $k=\omega /\left[c\left(1-\mathrm{i} \xi_{T}\right)\right]$ where the damping $\xi_{T}=5.8 \%$ has been retrieved from measurements in the tube with the rigid termination. The pressure $p_{T}(x)$ reads:

$$
\frac{p_{T}(x)}{\rho_{e} c v_{s}}=\frac{\cos \left(k\left(x-x_{f}\right)\right)-\mathrm{i} \rho_{e} c \Upsilon \sin \left(k\left(x-x_{\S}\right)\right)}{\mathrm{i} \sin \left(k\left(H-x_{f}\right)\right)-\rho_{e} c \Upsilon \cos \left(k\left(H-x_{f}\right)\right)} .
$$

The model predictions for the resonant surface and the rigid termination are compared with the data in Fig. 11. The resonance frequency of the tube with the rigid termination is $359 \mathrm{~Hz}$. However, in the presence of the resonators, an antiresonance occurs at this frequency with pressure being reduced by nearly a factor of 10 , that is $\left|p_{T} /\left(\rho_{e} c v_{s}\right)\right| \approx 0.5$ instead of $\approx 4.9$ with the rigid end. The coupled system exhibits two resonance peaks, in accordance with the model predictions.

\section{Anechoic chamber measurements-nearly total sound absorption by a finite array}

As shown in the previous section, the nearly total absorption of sound at resonance has been observed in the impedance tube where the idealised conditions of normally incident plane wave and emulated infinite surface have been achieved. In this section the robustness of this effect is investigated by performing measurements in anechoic conditions using a pointsource excitation and a finite size resonant surface.

\subsection{Experimental setup}

The experimental setup is illustrated in Fig. 12. It consists of a square lattice arrangement of $23 \times 23=529$ identical resonators on a square rigid board $(122 \mathrm{~cm}$-wide, $2.5 \mathrm{~cm}$-thick medium density fibreboard), excited by a speaker (Genelec, type 1029A) hung $157 \mathrm{~cm}$ above the centre of the board and facing it. It has been checked experimentally that the speaker acts as a monopole source located at $(x, y, z)=(-5.8,0,157) \mathrm{cm}$ in the frequency range below $500 \mathrm{~Hz}$.

The resonators are similar to those of configuration $C$ in Table 1 . They have been characterised individually in an impedance tube. Fig. 13 shows the cumulative distribution of the resonance frequency $\omega_{0} /(2 \pi)$ and loss factor $\zeta / \omega_{0}$ 
a

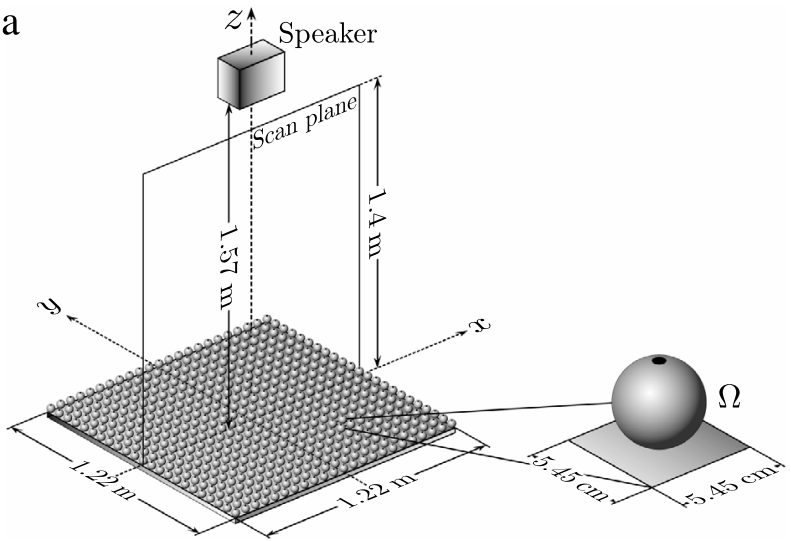

b

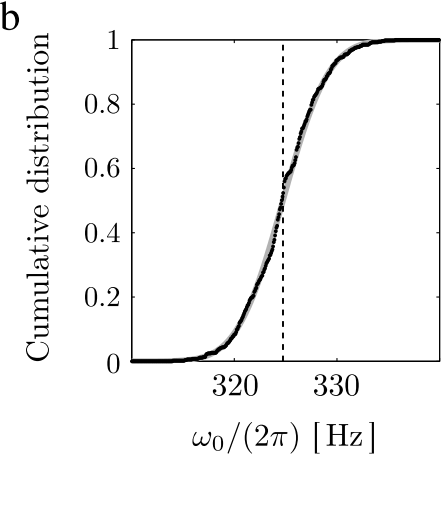

c

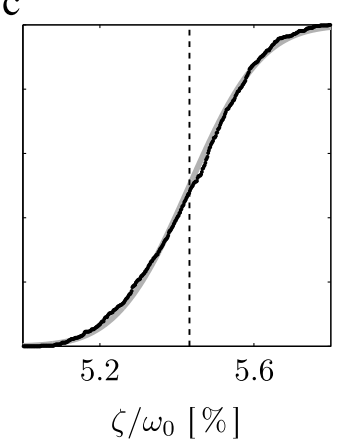

Fig. 12. (a) Experimental set-up used for measurements in anechoic chamber. (b) and (c) Cumulative distribution of the resonance frequency and the loss factor of the resonators used in anechoic chamber measurements. Comparison with normal distribution (grey line) with the following mean values and standard deviations: frequency: $325 \mathrm{~Hz} \pm 3.5 \mathrm{~Hz}$; and loss factor $5.43 \% \pm 0.15 \%$.

measured. They satisfy the normal distributions having the following mean values and standard deviations: $325 \mathrm{~Hz} \pm 3.5 \mathrm{~Hz}$ for the resonance frequency and $5.43 \% \pm 0.15 \%$ for the loss factor. This confirms that the resonators used in the experiment are very similar to each other and that the array can be considered as periodic with a very good approximation. The measured resonance frequency and loss factor are in good agreement with the following theoretical estimates, $\omega_{0} /(2 \pi)=349 \mathrm{~Hz}$ and $\zeta / \omega_{0}=4.5 \%$, and with the results shown in Fig. 7. The lattice constant $\ell$ of the resonant surface has been chosen so that the ratio $\eta / \zeta$ with a single resonator upon $\Sigma$ is the same as that, $\eta_{T} / \zeta_{T}$, in configuration $C$ with three resonators in the impedance tube. That leads to $3 /\left(\zeta_{T}\left|\Sigma_{T}\right|\right)=1 /\left(\zeta \ell^{2}\right)$, with $\zeta_{T} \approx 6 \%$ and $\left|\Sigma_{T}\right|=\pi D_{T}^{2} / 4$, which results in $\ell \approx 5.4 \mathrm{~cm}$ and a scale parameter $\epsilon_{0}=\ell \omega_{0} / c \approx 0.32$ that indicates an actual scale separation around the resonance frequency.

The pressure field $p$ have been measured in the $x z$ plane above the resonant surface (for $x \in[-60 ; 60] \mathrm{cm}$ and $z \in[0 ; 140] \mathrm{cm}$ every $20 \mathrm{~cm})$ and in the cavity of central resonator at $(x, y)=(0,0)$. To measure the pressure field in the resonator cavity, a quarter-inch-diameter hole has been drilled in it, and the head of a quarter-inch pressure microphone has been inserted in the hole and sealed to avoid leakages. Measurements have been performed with the speaker alone, so as to measure the incident field $p_{\text {inc }}$, and in the presence of the rigid board with and without the resonators on it (pressure fields $p^{\text {res }}$ and $p^{\text {rig }}$ respectively). The input signal fed to the speaker was a Ricker wavelet (input voltage in the form $\left.V_{\text {in }}(t) \propto\left[\omega_{c}^{2} / 2-1\right] \exp \left[-\omega_{c}^{2} t^{2} / 4\right]\right)$ with central frequency $\omega_{c} /(2 \pi)$ close to the resonance frequency $325 \mathrm{~Hz}$.

\subsection{Evidence of nearly total sound absorption}

In Fig. 13(a) the amplitude of pressure $p_{r}^{\text {res }}=p^{\text {res }}-p_{\text {inc }}$ reflected by the resonant surface is shown, normalised to that reflected from the rigid board, $p_{r}^{\text {rig }}=p^{\text {rig }}-p_{\text {inc }}$, at three different heights $z=\{40,60,80\} \mathrm{cm}$ above the centre of the board. While $p_{r}^{\text {res }} \approx p_{r}^{\text {rig }}$ at frequencies lower $\omega \ll \omega_{0}$ and higher $\omega \gg \omega_{0}$ than the resonance frequency $\omega_{0} /(2 \pi)$, the measurements show a nearly total absorption of the incident field at the resonance, with $p_{r}^{\text {res }} \rightarrow 0$ for all three heights. Experimental results are in a good agreement with the reflection coefficient predictions assuming plane waves, despite the point source excitation and the finite size of the board. Fig. 13(b) presents the amplitude of pressure $p_{0}$ inside the cavity of the central resonator, normalised to that $p_{s}$ measured at the surface next to the resonator, at $(x, y, z) \approx(-2.7,0,0) \mathrm{cm}$. As discussed in Section 3.2, it shows the amplification of the pressure in the cavity at resonance, when the field radiated by the resonant surface is nearly eliminated due to the losses. This provides an experimental evidence of the spatial localisation of the incident energy within the resonators at the resonance. Experimental results are in a good agreement with the theoretical transfer function $p_{o} / p_{s}=\omega_{0}^{2} /\left[\omega_{0}^{2}-\mathrm{i} \zeta \omega-\omega^{2}\right]$ of the Helmholtz resonator.

The real part of the transfer function between the pressure field $p$ and the input voltage $V_{\text {in }}$ is mapped in the $(x, z)$ plane above the surface for two frequencies in Fig. 14. Physically, that corresponds to the pressure field that would be measured if a sinusoidal signal with unitary amplitude were fed to the speaker. Maps are shown in the free field (speaker alone) and in the presence of the rigid board with and without the resonators on it. Fig. 14(a) shows results at the resonance frequency $325 \mathrm{~Hz}$. While the field amplification around $z=50 \mathrm{~cm}$ and the complex wavefronts above the surface are observed above the rigid board due to the interference between the incident and the reflected waves, the reflection-free spherical wavefronts of the incident field are recovered when the resonators are arranged on it. That testifies the nearly total absorption of the incident field at resonance by the resonant surface. By contrast, Fig. 14(b) shows that, at the frequency of $250 \mathrm{~Hz}$ which is below the resonance, the pressure distribution above the rigid and the resonant surfaces are very similar. This illustrates the fact that, outside the vicinity of the resonance, the resonant surface behaves as if it were rigid, with a negligible absorption of the incident field. 

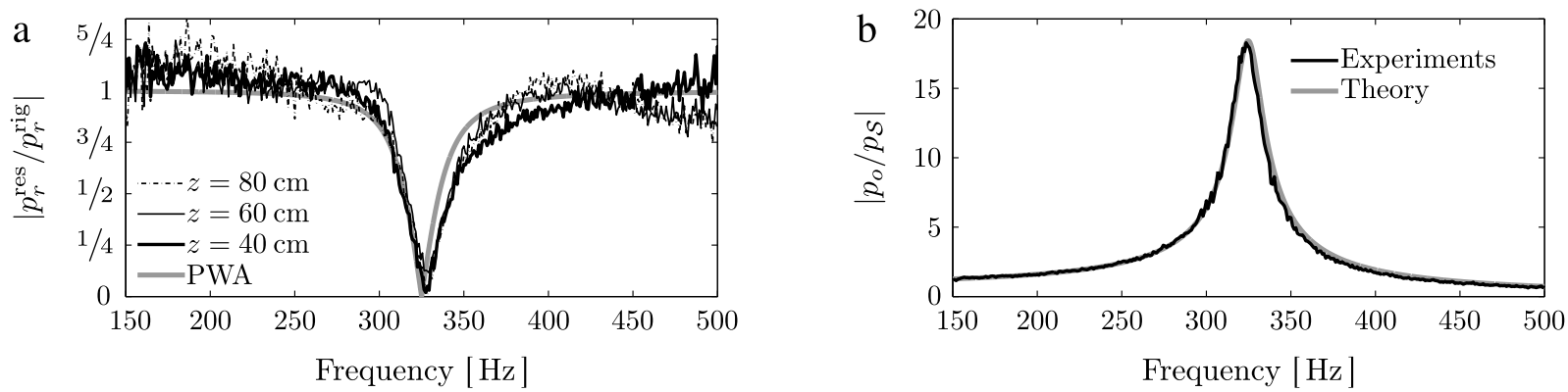

Fig. 13. Transfer functions. (a) Amplitude of pressure reflected by the resonant surface normalised to that reflected by the rigid board; (b) amplitude of pressure inside the resonator cavity normalised to that on the surface next to the resonator. Experimental results compared with (a) admittance model assuming plane waves (PWA) using resonators' parameters deduced from characterisation tests; and (b) the Helmholtz resonator transfer function.
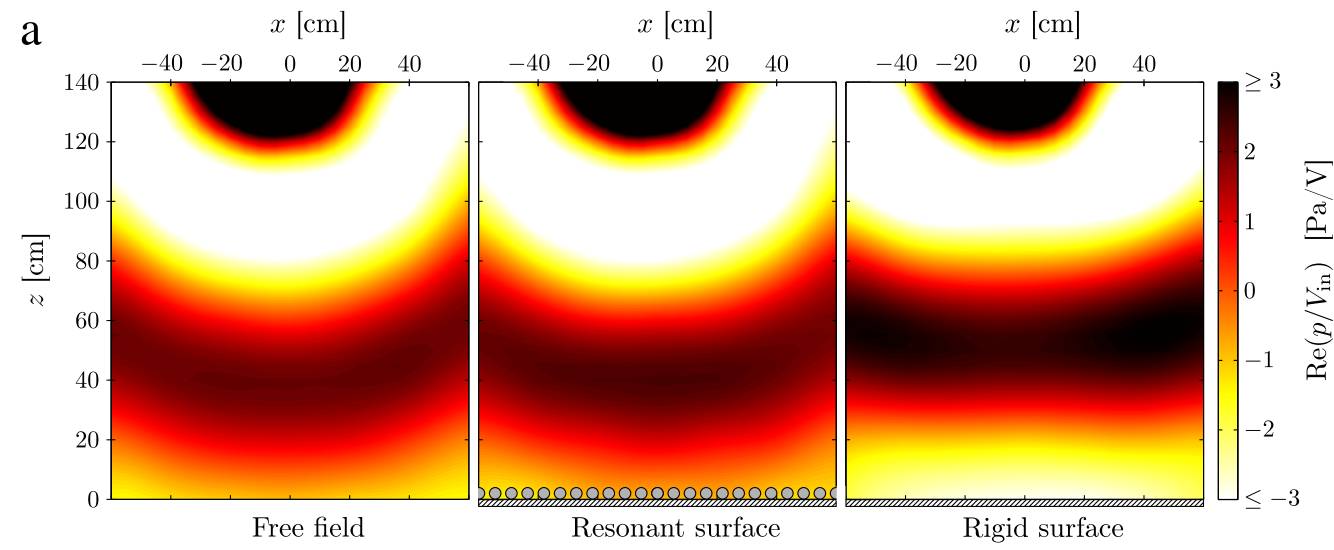

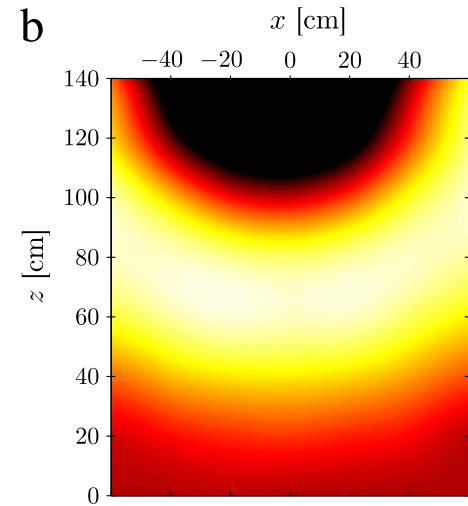

Free field

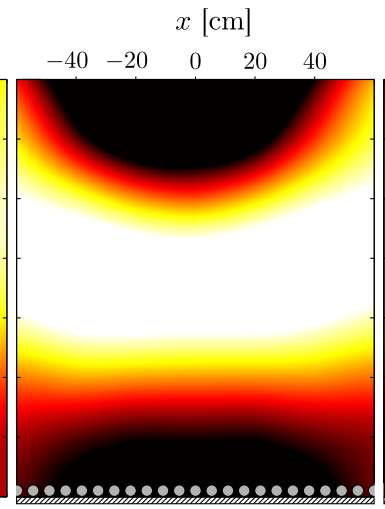

Resonant surface

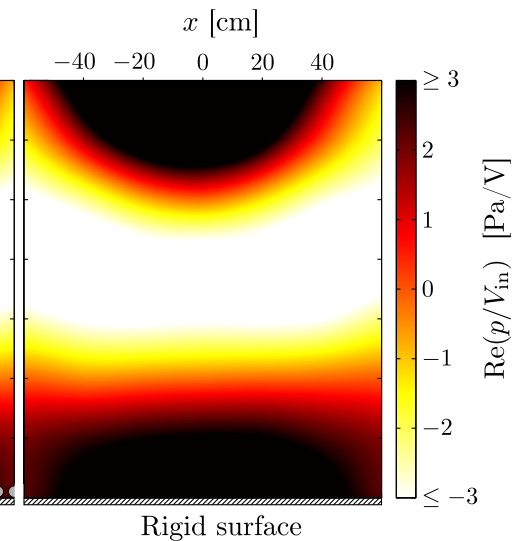

Rigid surface

Fig. 14. Real part of the measured transfer function between the pressure field and the input voltage fed to the speaker: (a) at the resonance frequency $325 \mathrm{~Hz}$; (b) at the frequency $250 \mathrm{~Hz}$. Experimental results shown in free field condition (speaker alone); with the resonant surface; and with the rigid board without the resonators (Colour online).

\section{Conclusions}

The coincidence within the same frequency range of the long wavelength dynamics of sound waves propagating in air with the subwavelength resonances occurring in the close vicinity of the boundary is the specific feature defining the acoustical properties of the resonant metasurfaces. Under the condition of scale separation for both in-plane and out-ofplane characteristic lengths, the two-scale asymptotic homogenisation model, based on the fundamental principles applied to the local scale, is shown to successfully predict the macroscopic behaviour of resonant surfaces. In particular, it provides a simple effective admittance formula suitable for fast computations and identifies the four design parameters: resonance frequency $\omega_{0}$, the scale separation parameter at the resonance $\epsilon_{0}=\ell \omega_{0} / c$, the admittance parameter $\eta=\rho_{e} c A^{2} /(M|\Sigma|)$ and the loss factor $\zeta / \omega_{0}$. The homogenisation model offers a general theoretical framework for linear resonators of any kind, whether they are upon, above or partially embedded in the supporting surface. According to the model, resonant surface 
effects are more related to the acoustic flux distribution the resonators create at the boundary than to their exact nature. In this paper the model has been applied to predict the total absorption of sound through deep subwavelength critical coupling, broadband absorption of sound, the phase reversal upon reflection (which is critical for the wavefront manipulation by graded arrays), enclosure mode control and memory effects related to the co-dynamics between the resonators and the long wavelength field. In the design of the resonant surface for the laboratory measurements, a particular attention has been paid to the construction of the resonators that satisfy the condition of scale separation. This resulted in the use of spherical Helmholtz resonators with extended inner necks. These cheap and easy-to-realise resonators have been used as the basic elements of the thin and light resonant surface, with the thickness $h 26$ times smaller than sound wavelength $\lambda_{0}$ at resonance, the lattice size $\ell$ nearly 20 times smaller than $\lambda_{0}$, the filling fraction, i.e., the fraction of the REV volume occupied by the resonator, $V /\left(\ell^{2} h\right) \approx 0.28$, and the added mass of $0.875 \mathrm{~kg} / \mathrm{m}^{2}$. The model predictions have been first validated in the experiments performed under the idealised conditions of the impedance tube where the incident wave is plane and the infinite surface is emulated. After that, the experiments have been performed in the anechoic chamber with a point source and the surface array of the finite size. A good agreement between the model and the data is demonstrated in both cases confirming the robustness of the surface design. With the array designed according to the theoretical principles, the regime of co-dynamics between the long wavelength field and the resonators has been observed in the experiments, in particular through the direct measurement of the local field inside the resonators. It is planned to apply this model to study the conditions for surface waves generation and to extend it to surface arrays with graded properties.

\section{Acknowledgements}

The authors are grateful to S. Taherzadeh and K. Attenborough for their active support of the project and to A. Elliott and C. Lomax for their help in the experiments. The research has been supported by the UK Engineering and Physical Sciences Research Council under grant agreement EP/K037234/1.

\section{Appendix A. Homogenisation}

\section{A.1. Two-scale description and Taylor expansions}

Given the existence of the two characteristic sizes $L$ and $\ell=\epsilon L$ in the system, the usual space variable $\mathbf{x}$ is normalised by either length to provide two dimensionless variables $\overline{\mathbf{x}}=\mathbf{x} / L$ and $\overline{\mathbf{y}}=\mathbf{x} / \ell=\epsilon^{-1} \overline{\mathbf{x}}$ and their respective del operators $\nabla_{\overline{\mathbf{x}}}$ and $\nabla_{\overline{\mathbf{y}}}$. As a result, differentiation is modified according to $\nabla=L^{-1}\left(\nabla_{\overline{\mathbf{x}}}+\epsilon^{-1} \nabla_{\overline{\mathbf{y}}}\right)$. This allows the description of the field variations at both scales, with $\overline{\mathbf{x}}$ and $\overline{\mathbf{y}}$ describing variations at the macroscopic and microscopic scale respectively. The macroscopic fields $P$ and $\mathbf{V}$ vary significantly over distances $\mathcal{O}(L)$. For this reason their spatial variations ate described by the variable $\overline{\mathbf{x}}$, that is $P(\overline{\mathbf{x}})$ and $\mathbf{V}(\overline{\mathbf{x}})$. The BL fields $p^{\star}$ and $\mathbf{v}^{\star}$ are locally $\Sigma$-periodic with the macroscopic modulation in the plane $\&$. Consequently, their dependence on the two normalised space variables is introduced $[11,12]$ in the form $p^{\star}\left(\overline{\mathbf{x}}_{\delta}, \overline{\mathbf{y}}\right)$ and $\mathbf{v}^{\star}\left(\overline{\mathbf{x}}_{s}, \overline{\mathbf{y}}\right)$, where the index \& denotes the projection on the plane $\&$. The BL fields are $\Sigma$-periodic and evanescent over $\overline{\mathbf{y}}$ while the first variable $\overline{\mathbf{x}}_{\S}$ is used to describe the in-plane modulation. The particle velocity $\mathbf{v}_{n}^{j}$ produced by the structure $j$ in the REV $\Omega_{n}$, and the vector $\mathbf{n}_{n}^{j}$ normal to the boundary $\Gamma_{n}^{j}$ of that structure, depend exclusively on the variable $\overline{\mathbf{y}}$, that is $\mathbf{v}_{n}^{j}(\overline{\mathbf{y}})$ and $\mathbf{n}_{n}^{j}(\overline{\mathbf{y}})$. Further, the macroscopic variations of the propagating fields $(P, \mathbf{V})$ and BL fields $\left(p^{\star}, \mathbf{v}^{\star}\right)$ are downscaled to each cell $\Omega_{n}$. Since a subwavelength distance $\mathcal{O}(\ell) \ll L$ can be considered as a small increment for the variable $\overline{\mathbf{x}}$, the macroscopic variations at any point $\mathbf{x}=L \overline{\mathbf{x}}=\ell \overline{\mathbf{y}}$ of the REV $\Omega_{n}$ can be deduced from those at the centroid position $\mathbf{x}_{n}=L \overline{\mathbf{x}}_{n}=\ell \overline{\mathbf{y}}_{n}=\sum_{j} \int_{\Gamma^{j}} \mathbf{x} d S /\left[\sum_{j}\left|\Gamma^{j}\right|\right]$ of $\Omega_{n}$ using Taylor expansions [31,32]. For instance, and similarly for $p^{\star}, \mathbf{V}$ and $\mathbf{v}^{\star}$ :

$$
P(\overline{\mathbf{x}})=P\left(\overline{\mathbf{x}}_{n}\right)+\epsilon\left[\operatorname{grad}_{\overline{\mathbf{x}}} P\right]_{\overline{\mathbf{x}}=\overline{\mathbf{x}}_{n}} \cdot\left(\overline{\mathbf{y}}-\overline{\mathbf{y}}_{n}\right)+\epsilon^{2} \ldots
$$

\section{A.2. Governing equations at the local scale}

The governing equations of mass and momentum conservation are written for the superposition of the propagating fields $(P, \mathbf{V})$ and the BL fields $\left(p^{\star}, \mathbf{v}^{\star}\right)$ with the two-scale differentiation:

$$
\frac{L i \omega}{\gamma P_{e}}\left(P+p^{\star}\right)=\left\{\operatorname{div}_{\overline{\mathbf{x}}}+\epsilon^{-1} \operatorname{div}_{\overline{\mathbf{y}}}\right\}\left(\mathbf{V}+\mathbf{v}^{\star}\right) ; \quad L i \omega \rho_{e}\left(\mathbf{V}+\mathbf{v}^{\star}\right)=\left\{\operatorname{grad}_{\overline{\mathbf{x}}}+\epsilon^{-1} \operatorname{grad}_{\overline{\mathbf{y}}}\right\}\left(P+p^{\star}\right) .
$$

Due to BL evanescence, the propagating field $(P(\overline{\mathbf{x}}), \mathbf{V}(\overline{\mathbf{x}}))$ prevails some distance away from the surface, where it satisfies the conservation equations on its own, expressed with the space variable $\overline{\mathbf{x}}$ :

$$
\frac{\operatorname{Li} \omega}{\gamma P_{e}} P(\overline{\mathbf{x}})=\operatorname{div}_{\overline{\mathbf{x}}} \mathbf{V}(\overline{\mathbf{x}}) ; \quad \operatorname{Li} \omega \rho_{e} \mathbf{V}(\overline{\mathbf{x}})=\operatorname{grad}_{\overline{\mathbf{x}}} P(\overline{\mathbf{x}})
$$


Subtracting (A.3) from (A.2) using the linearity of the problem, the BL field $\left(p^{\star}, \mathbf{v}^{\star}\right)$ is found to satisfy the following equations of mass and momentum conservation:

$$
\frac{\operatorname{Li} \omega}{\gamma P_{e}} p^{\star}=\left\{\operatorname{div}_{\overline{\mathbf{x}}}+\epsilon^{-1} \operatorname{div}_{\overline{\mathbf{y}}}\right\} \mathbf{v}^{\star} ; \quad \operatorname{Li} \omega \rho_{e} \mathbf{v}^{\star}=\left\{\operatorname{grad}_{\overline{\mathbf{x}}}+\epsilon^{-1} \operatorname{grad}_{\overline{\mathbf{y}}}\right\} p^{\star} .
$$

Besides, the boundary conditions at the surface $\Gamma_{n}^{j}$ of the structure $j$ in the REV $\Omega_{n}$, that is the resonators $j \in \llbracket 1, N \rrbracket$ and the rigid substrate $j=0$, take the form:

$$
\mathbf{v}_{n}^{j}=\mathcal{R}^{j}\left(P+p^{\star}\right) \text { and }\left(\mathbf{V}+\mathbf{v}^{\star}\right) \cdot \mathbf{n}_{n}^{j}=\mathbf{v}_{n}^{j} \cdot \mathbf{n}_{n}^{j} \text { at } \Gamma_{n}^{j} .
$$

Note that the spacing between the structures within the REV is supposed to be significantly larger than viscous and thermal skin depths. This allows neglecting viscous and thermal losses in air.

\section{A.3. Asymptotic expansions and problem at the leading order}

The resonant surface is assumed to play a significant role in the mass balance, for this reason all particle velocities are set to be of the same order, i.e. $\mathbf{v}^{\star}=\mathcal{O}(\mathbf{V})=\mathcal{O}\left(\mathbf{v}_{n}^{j}\right)$. Next, all unknown fields are expanded asymptotically in powers of $\epsilon \ll 1$. For instance, for the pressure in the BL this expansion is

$$
p^{\star}\left(\overline{\mathbf{x}}_{\delta}, \overline{\mathbf{y}}\right)=p^{\star(0)}\left(\overline{\mathbf{x}}_{\delta}, \overline{\mathbf{y}}\right)+\epsilon p^{\star(1)}\left(\overline{\mathbf{x}}_{\delta}, \overline{\mathbf{y}}\right)+\epsilon^{2} \ldots
$$

where the order of the terms is indicated by the bracketed superscripts. Similar expansions are performed for $P, \mathbf{V}, \mathbf{v}^{\star}$ and $\mathbf{v}_{n}^{j}$. These asymptotic expansions are then substituted in Eqs. (A.1)-(A.5). Terms of equal powers of $\epsilon$ are collected, which results in a series of problems that can be solved successively in increasing order of powers of $\epsilon$. At the leading order (power $\left.\epsilon^{0}\right)$, the following problem holds:

$$
\begin{aligned}
& \operatorname{grad}_{\overline{\mathbf{y}}} p^{\star(0)}=\mathbf{0} ; \\
& \operatorname{div}_{\overline{\mathbf{y}}} \mathbf{v}^{\star(0)}=0 ; \\
& \left(\mathbf{V}^{(0)}+\mathbf{v}^{\star(0)}\right) \cdot \mathbf{n}_{n}^{j}=\mathbf{v}_{n}^{j(0)} \cdot \mathbf{n}_{n}^{j} \quad \text { on } \Gamma_{n}^{j} ; \\
& \mathbf{v}_{n}^{j(0)}=\mathcal{R}^{j}\left[P^{(0)}+p^{\star(0)}\right] \quad \text { on } \Gamma_{n}^{j} ; \\
& \mathbf{v}^{\star(0)} \rightarrow \mathbf{0} \text { and } \quad p^{\star(0)} \rightarrow 0 \quad \text { as } \overline{\mathbf{y}} \cdot \mathbf{n} \rightarrow \infty \\
& P^{(0)}\left(\overline{\mathbf{x}} \in \Omega_{n}\right)=P^{(0)}\left(\overline{\mathbf{x}}_{n}\right) ; \\
& \mathbf{V}^{(0)}\left(\overline{\mathbf{x}}^{(0)} \Omega_{n}\right)=\mathbf{V}^{(0)}\left(\overline{\mathbf{x}}_{n}\right) ; \\
& p^{\star(0)}\left(\overline{\mathbf{x}}_{f}, \overline{\mathbf{y}}\right)=p^{\star(0)}\left(\overline{\mathbf{x}}_{n}, \overline{\mathbf{y}}\right) ; \\
& \mathbf{v}^{\star(0)}\left(\overline{\mathbf{x}}_{s}, \overline{\mathbf{y}}\right)=\mathbf{v}^{\star(0)}\left(\overline{\mathbf{x}}_{n}, \overline{\mathbf{y}}\right) .
\end{aligned}
$$

Eq. (A.7a) shows that BL pressure $p^{\star(0)}$ is independent on the local spatial variable $\overline{\mathbf{y}}$. Consequently it is uniform on $\Omega_{n}$ and is equal to zero due to the evanescence condition (A.7e). Eq. (A.7b) of the BL incompressibility is integrated with respect to $\overline{\mathbf{y}}$ over the column $\mathcal{C}_{n}$ of air inside and above $\Omega_{n}$, see Fig. 2 . The boundary $\partial \mathcal{C}_{n}$ of the column $\mathcal{C}_{n}$ consists of the rigid backing, the surface $\Gamma_{n}^{j}$ of the structures, its cross-section $\Sigma$ located at $\overline{\mathbf{y}} \cdot \mathbf{n} \rightarrow \infty$ and its lateral surface $\Gamma_{\text {lat }}$. Applying the divergence theorem and using the BL $\Sigma$-periodicity and evanescence, the following equation is derived:

$$
\int_{\mathfrak{C}_{n}} \operatorname{div}_{\overline{\mathbf{y}}} \mathbf{v}^{\star(0)} d \Omega_{\overline{\mathbf{y}}}=-\sum_{j \in \mathbb{0} ; N \rrbracket} \int_{\Gamma_{n}^{j}} \mathbf{v}^{\star(0)} \cdot \mathbf{n}_{n}^{j} d \Gamma_{\overline{\mathbf{y}}}=0 .
$$

Further, since $\mathbf{V}^{(0)}$ is uniform at the local scale, see Eq. (A.7g), the following relation also holds:

$$
-\sum_{j \in \llbracket 0 ; N \rrbracket} \int_{\Gamma_{n}^{j}} \mathbf{V}^{(0)} \cdot \mathbf{n}_{n}^{j} d \Gamma_{\overline{\mathbf{y}}}+|\Sigma|_{\overline{\mathbf{y}}} \mathbf{V}^{(0)}\left(\overline{\mathbf{x}}_{n}\right) \cdot \mathbf{n}=0
$$

where $|\Sigma|_{\overline{\mathbf{y}}}=\int_{\Sigma} d \Sigma_{\overline{\mathbf{y}}}$. The term $|\Sigma|_{\overline{\mathbf{y}}} \mathbf{n} \cdot \mathbf{V}^{(0)}$ results from the flux through the section of $\mathcal{C}_{n}$ located at $\overline{\mathbf{y}} \cdot \mathbf{n} \rightarrow \infty$. Eq. (A.7c) is now integrated over the boundary $\Gamma_{n}^{j}$, providing the following balance of flux:

$$
\int_{\Gamma_{n}^{j}}\left(\mathbf{V}^{(0)}+\mathbf{v}^{\star(0)}\right) \cdot \mathbf{n}_{n}^{j} d \Gamma_{\overline{\mathbf{y}}}=\int_{\Gamma_{n}^{j}} \mathbf{v}_{n}^{j(0)} \cdot \mathbf{n}_{n}^{j} d \Gamma_{\overline{\mathbf{y}}} .
$$

Combining Eqs. (A.8)-(A.10), with $\mathbf{v}_{n}^{(0)} \cdot \mathbf{n}_{n}^{0}=0$ at the rigid substrate $(j=0$ ), the mass conservation law at the boundary is found for the macroscopic field:

$$
\mathbf{V}^{(0)}\left(\overline{\mathbf{x}}_{n}\right) \cdot \mathbf{n}=\sum_{j \in \llbracket 1 ; N \rrbracket} \frac{1}{|\Sigma|_{\overline{\mathbf{y}}}} \int_{\Gamma_{n}^{j}} \mathbf{v}_{n}^{j(0)} \cdot \mathbf{n}_{n}^{j} d \Gamma_{\overline{\mathbf{y}}}
$$


Rewriting (A.11) using the usual space variable $\mathbf{x}$, Eq. (1) is derived. If a sharp scale separation is achieved, the leading order description (power $\epsilon^{0}$ ) is sufficient to provide a reasonably accurate description of the propagating field. Otherwise, corrective terms of order $\epsilon, \epsilon^{2}$, etc. in the asymptotic expansions need to be considered.

\section{A.4. Impedance tube characterisation}

Eqs. (A.8)-(A.11) still hold if rigid boundary conditions $\mathbf{V} \cdot \mathbf{n}=0$ and $\mathbf{v}^{\star} \cdot \mathbf{n}=0$ are prescribed at the lateral surface $\Gamma_{\text {lat }}$ of the column $\mathcal{C}_{n}$ instead of periodicity conditions: in the former case, the flux through $\Gamma_{\text {lat }}$ is equal to zero, while, in the latter case, the flux is self-balanced due to periodicity. As a result, the mass law (A.11) and, consequently, the effective admittance condition (3) remain valid at the leading order if the column $\mathcal{C}_{n}$ is, in fact, an impedance tube in which a unit cell of the resonant surface is arranged. In this case, the cross-section of the impedance plays the role of the period $\Sigma$.

\section{Appendix B. Admittance of other types of resonators}

In this Appendix, the admittance $Y$ of the quarter-wavelength resonator and the air-filled elastic shell. For simplicity, the analysis is performed here for lossless resonators but the dissipation can be introduced a posteriori, as explained for the case of the quarter-wavelength resonator.

\section{B.1. Quarter-wavelength resonator}

Consider the resonator made of the straight tube with the length $h$, and circular cross section $A$ of radius $a$, and denote $z$ the coordinate along the tube axis. The pressure field $p_{o}(z)$ and the outward axial particle displacement $u_{o}(z)$ in the resonator are governed by the following equations of momentum and mass conservation:

$$
-\omega^{2} \rho_{e} u_{0}=-\frac{\partial p_{o}}{\partial z} ; \quad p_{o}=-\gamma P_{e} \frac{\partial u_{o}}{\partial z} .
$$

The resonator is connected to the outer space at the aperture $z=0$ where the macroscopic pressure field $P$ is prescribed, while the tube is closed at the other end, that is $u_{0}=0$ at $z=-h$. The modal analysis of this problem, performed with $P=0$, results in the eigenfrequencies $\omega_{m}=(2 m+1) \omega_{0}$, where $\omega_{0}=\pi c / 2 h$ and $m \geq 0$ is an integer, and in the modal displacement $\varphi_{m}(z)=\cos \left(\omega_{m} z / c\right)$. Consider now the weak formulation of the momentum conservation, with the displacement $\hat{u}$ as the test-field:

$$
-\omega^{2} \rho_{e} \int_{z=-h}^{z=0} u_{0} \hat{u} d z=-\int_{z=-h}^{z=0} \frac{\partial p_{o}}{\partial z} \hat{u} d z
$$

Integrating the right-hand side by parts, applying the boundary conditions, and using the mass conservation yield:

$$
\gamma P_{e} \int_{z=-h}^{z=0} \frac{\partial \hat{u}}{\partial z} \frac{\partial u_{o}}{\partial z} d z-\omega^{2} \rho_{e} \int_{z=-h}^{z=0} u_{o} \hat{u} d z=-P \hat{u}(0) .
$$

This equation is in the form of Eq. (4). Following the same procedure as in Section 2.4 and using the orthogonality of the modes, the displacement $u_{o}$ is found in the form:

$$
u_{o}(z)=-P \sum_{m} \frac{A / M_{m}}{\omega_{m}^{2}-\omega^{2}} \varphi_{m}(z),
$$

where the modal mass is $M_{m}=\rho_{e} S \int_{z=-h}^{z=0} \varphi_{m}^{2} d z=M / 2$, with $M=\rho_{e} A h$ being the mass of air in the tube. Finally, the resonator admittance $Y=-\mathrm{i} \omega A u_{0}(0) / P$ is given by:

$$
Y=\mathrm{i} \omega \sum_{m} \frac{2 A^{2} / M}{\omega_{m}^{2}-\omega^{2}} .
$$

The weak damping in the resonator can be taken into account by introducing the dissipation terms $-\mathrm{i} \zeta_{m} \omega$ at the denominators of the admittance $Y$, see Eq. (7), where the loss factors $\zeta_{m} / \omega_{m} \ll 1$ can be found from characterisation tests, see Section 4.2. Note that, for the first resonance to occur under the condition of scale separation, that is $\epsilon_{0}=\ell \omega_{0} / c \ll 1$, the following design constraint must be satisfied: $h / \ell=\epsilon_{0}^{-1} \pi / 2$, that is, the tube length must be one order larger than the size of the REV. If the tube of the quarter-wavelength resonator is folded so as to obtain a bulky shape that fits in the REV, the following condition must hold also: $A h \leq \mathcal{O}(|\Sigma| \ell)$. Combining the two relations yields $A \leq \mathcal{O}\left(\epsilon_{o}|\Sigma|\right)$, that is, the tube cross section must be (at least) one order smaller than the area $|\Sigma|$ of the array period. Similarly to Helmholtz resonators, viscous and thermal losses are stronger in narrow tubes, see Section 4.1, which limits the miniaturisation of the quarter-wavelength resonator for deep-subwavelength applications. 


\section{B.2. Air-filled elastic shell}

Consider an elastic shell $\mathscr{E}$ with air inside. For the elastic shell, the displacement $\mathbf{u}$ and the stress tensor $\sigma$ satisfy the following equations of dynamic equilibrium and stress/strain relationship, with the density $\rho$ and the elastic tensor $\mathcal{C}$ :

$$
\operatorname{div}(\boldsymbol{\sigma})=-\rho \omega^{2} \mathbf{u} ; \quad \boldsymbol{\sigma}=\mathcal{C}: \mathbf{e}(\mathbf{u}),
$$

where $\mathbf{e}(\mathbf{u})=\left(\operatorname{grad}(\mathbf{u})+{ }^{T} \operatorname{grad}(\mathbf{u})\right) / 2$ is the strain tensor. In the enclosure $\mathcal{V}_{\text {in }}$ formed by the shell, air is at the quasistatic equilibrium, which results in the (quasi-)uniform pressure field $p_{\text {in }}$, while the particle displacement $\mathbf{u}_{\text {in }}$ satisfies the following equation of mass conservation:

$$
p_{\text {in }}=-\gamma P_{e} \operatorname{div}\left(\mathbf{u}_{\text {in }}\right) \text {. }
$$

At the outer boundary $\Gamma$ (outward normal vector $\mathbf{n}$ ), the uniform macroscopic pressure $P$ is prescribed, while at the inner boundary $\Gamma_{\text {in }}$ (normal vector $\mathbf{n}^{\prime}$ directed at the enclosure), the conditions of surface stress and normal displacement continuity hold. These boundary conditions take the form:

$$
\boldsymbol{\sigma} \cdot \mathbf{n}=-P \mathbf{n} \text { at } \Gamma ; \quad \boldsymbol{\sigma} \cdot \mathbf{n}^{\prime}=-p_{\text {in }} \mathbf{n}^{\prime} \text { at } \Gamma_{\text {in }} ; \quad \mathbf{u} \cdot \mathbf{n}^{\prime}=\mathbf{u}_{\text {in }} \cdot \mathbf{n}^{\prime} \text { at } \Gamma_{\text {in }} .
$$

First, integrate the mass conservation Eq. (B.7) over the volume $\mathcal{V}_{\text {in }}$ of the enclosure. Using the divergence theorem and the continuity of normal displacement at $\Gamma_{\text {in }}$, this yields:

$$
\left|\mathcal{V}_{\text {in }}\right| p_{\text {in }}=\gamma P_{e} \int_{\Gamma_{\text {in }}} \mathbf{u}_{\text {in }} \cdot \mathbf{n}^{\prime} d \Gamma=\gamma P_{e} \int_{\Gamma_{\text {in }}} \mathbf{u} \cdot \mathbf{n}^{\prime} d \Gamma .
$$

Now, consider the weak formulation of the dynamic equilibrium of the elastic shell. Using $\hat{\mathbf{u}}$ as the test field, this leads to:

$$
\int_{\mathcal{E}} \boldsymbol{\sigma}: \mathbf{e}(\hat{\mathbf{u}}) d \mathcal{E}-\rho \omega^{2} \int_{\mathcal{E}} \mathbf{u} \cdot \hat{\mathbf{u}} d \mathcal{E} .=\int_{\Gamma} \hat{\mathbf{u}} \cdot[\boldsymbol{\sigma} \cdot \mathbf{n}] d \Gamma+\int_{\Gamma_{\mathrm{in}}} \hat{\mathbf{u}} \cdot\left[\boldsymbol{\sigma} \cdot \mathbf{n}^{\prime}\right] d \Gamma .
$$

Using the boundary conditions and the stress/strain relationship in the shell, Eq. (B.10) takes the form:

$$
\int_{\mathcal{E}} \mathbf{e}(\mathbf{u}): \mathcal{C}: \mathbf{e}(\hat{\mathbf{u}}) d \mathcal{E}-\rho \omega^{2} \int_{\mathcal{E}} \mathbf{u} \cdot \hat{\mathbf{u}} d \mathcal{E}+p_{\text {in }} \int_{\Gamma_{\mathrm{in}}} \hat{\mathbf{u}} \cdot \mathbf{n}^{\prime} d \Gamma=-P \int_{\Gamma} \hat{\mathbf{u}} \cdot \mathbf{n} d \Gamma
$$

Finally, using Eq. (B.9), Eq. (B.10) can be written in the same form as Eq. (4), that is:

$$
\mathcal{K}(\mathbf{u}, \hat{\mathbf{u}})-\omega^{2} \mathcal{M}(\mathbf{u}, \hat{\mathbf{u}})=-P \int_{\Gamma} \hat{\mathbf{u}} \cdot \mathbf{n} d \Gamma,
$$

where

$$
\mathcal{K}(\mathbf{u}, \hat{\mathbf{u}})=\int_{\mathcal{E}} \mathbf{e}(\mathbf{u}): \mathcal{C}: \mathbf{e}(\hat{\mathbf{u}}) d \mathcal{E}+\frac{\gamma P_{e}}{\left|\mathcal{V}_{\text {in }}\right|} \int_{\Gamma_{\text {in }}} \mathbf{u} \cdot \mathbf{n}^{\prime} d \Gamma \int_{\Gamma_{\mathrm{in}}} \hat{\mathbf{u}} \cdot \mathbf{n}^{\prime} d \Gamma
$$

and

$$
\mathcal{M}(\mathbf{u}, \hat{\mathbf{u}})=\rho \int_{\mathcal{E}} \mathbf{u} \cdot \hat{\mathbf{u}} d \mathcal{E} .
$$

The same modal analysis as that presented in Section 2.4 can then be performed with these operators to derive the resonator admittance.

\section{References}

[1] A. Khelif, Y. Achaoui, S. Benchabane, V. Laude, B. Aoubiza, Locally resonant surface acoustic wave band gaps in a two-dimensional phononic crystal of pillars on a surface, Phys. Rev. B: Condens. Matter 81 (2010) 214303.

[2] L. Schwan, C. Boutin, Unconventional wave reflection due to "resonant surface", Wave Motion 50 (2013) $852-868$

[3] C. Boutin, L. Schwan, M.S. Dietz, Elastodynamic metasurface: Depolarization of mechanical waves and time effects, J. Appl. Phys. 117 (2015) 064902.

[4] Y. Ra'di, C.R. Simovski, S.A. Tretyakov, Thin perfect absorbers for electromagnetic waves: theory, design, and realizations, Phys. Rev. Appl. 3 (2015) 037001

[5] G. Ma, M. Yang, S. Xiao, P. Sheng, Acoustic metasurface with hybrid resonances, Nature Mater. 13 (2014) 873-878.

[6] V.L. Jordan, The application of Helmholtz resonators to sound-absorbing structures, J. Acoust. Soc. Am. 19 (1947) $972-981$.

[7] Y. Li, B. Liang, Z. Ming Gu, X. Ye Zou, J. Chun Cheng, Reflected wavefront manipulation based on ultrathin planar acoustic metasurfaces, Sci. Rep. 3 (2013) 02546.

[8] M.R. Schroeder, Binaural dissimilarity and optimum ceilings for concert halls: More lateral sound diffusion, J. Acoust. Soc. Am. 65 (1979) $958-963$.

[9] H. Chen, S. Zhai, C. Ding, S. Liu, C. Luo, X. Zhao, Meta-atom cluster acoustic metamaterial with broadband negative effective mass density, J. Appl. Phys. 115 (2014) 054905.

[10] A. Bensoussan, J.-L. Lions, G. Papanicolaou, Asymptotic Analysis for Periodic Structures, North-Holland Publishing Company, 1978.

[11] E. Sánchez-Palencia, in: E. Berlin (Ed.), Non-Homogeneous Media and Vibration Theory, Springer-Verlag, 1980.

[12] J.-L. Auriault, C. Boutin, C. Geindreau, Homogenization of Coupled Phenomena in Heterogenous Media, ISTE Ltd and John Wiley \& Sons, Inc., 2009.

[13] F.J. Sabina, J.R. Willis, Scattering of SH waves by a rough half-space of arbitrary slope, Geophys. J. R. Astron. Soc. 42 (1975) 685-703. 
[14] C.L. Holloway, E.F. Kuester, Equivalent boundary conditions for a perfectly conducting periodic surface with a cover layer, Radio Sci. 35 (2000) 661-681.

[15] C. Boutin, P. Roussillon, Assessment of the urbanization effect on seismic response, Bull. Seismol. Soc. Amer. 94 (2004) $251-268$.

[16] C. Boutin, P. Roussillon, Wave propagation in presence of oscillators on the free surface, Internat. J. Engrg. Sci. 44 (2006) $180-204$.

[17] B. Delourme, H. Haddar, P. Joly, Approximate models for wave propagation across thin periodic interfaces, J. Math. Pures Appl. 98 (2012) $28-71$.

[18] J.-J. Marigo, A. Maurel, Homogenization models for thin rigid structured surfaces and films, J. Acoust. Soc. Am. 140 (2016) $260-273$.

[19] J.-J. Marigo, A. Maurel, Two-scale homogenization to determine effective parameters of thin metallic-structured films, Proc. R. Soc. Lond. Ser. A Math. Phys. Eng. Sci. 472 (2012) 20160068

[20] L. Schwan, C. Boutin, L.A. Padrón, M.S. Dietz, P.-Y. Bard, C. Taylor, Site-city interaction: theoretical, numerical and experimental crossed-analysis, Geophys. J. Int. 205 (2016) 1006-1031.

[21] C. Boutin, Acoustics of porous media with inner resonators, J. Acoust. Soc. Am. 134 (2013) 4717-4729.

[22] C. Boutin, F.X. Bécot, Theory and experiments on poro-acoustics with inner resonators, Wave Motion 54 (2015) 76-99.

[23] J.-L. Auriault, G. Bonnet, Dynamique des composites élastiques périodiques, Arch. Mech. 37 (1985) 269-284.

[24] J.-L. Auriault, Acoustics of heterogeneous media: macroscopic behavior by homogenization, Curr. Top. Acoust. Res. 1 (1994) 63-90.

[25] Z. Liu, X. Zhang, Y. Mao, Y.Y. Zhu, Z. Yang, C.T. Chan, P. Sheng, Locally resonant sonic materials, Science 289 (2000) 1734-1736.

[26] N. Fang, D. Xi, J. Xu, M. Ambati, W. Srituravanich, C. Sun, X. Zhang, Ultrasonic metamaterials with negative modulus, Nature Mater. 5 (2006) $452-456$.

[27] M.A. Biot, Reflection on a rough surface from an acoustic point source, J. Acoust. Soc. Am. 29 (1957) 1193-1200.

[28] I. Bashir, S. Taherzadeh, K. Attenborough, Diffraction assisted rough ground effect: Models and data, J. Acoust. Soc. Am. 133 (2013) 1281-1292.

[29] P.M. Morse, K.U. Ingard, Theoretical Acoustics, McGraw-Hill, Inc., 1968.

[30] D. Caillerie, P. Trompette, P. Verna, Homogenisation of periodic trusses, in: Congres IASS, Madrid, 1989, pp. 7139-7180.

[31] G. Moreau, D. Caillerie, Continuum modeling of lattice structures in large displacement applications to buckling analysis, Comput. Struct. 68 (1998) $181-189$.

[32] C. Boutin, S. Hans, Homogenisation of periodic discrete medium: Application to dynamics of framed structures, Comput. Geotech. 30 (2003) 303-320.

[33] S. Forest, F. Pradel, K. Sab, Asymptotic analysis of heterogeneous Cosserat media, Internat. J. Engrg. Sci. 38 (2001) 4585-4608.

[34] J.-L. Auriault, C. Boutin, Deformable porous media with double porosity. Quasi-statics. I: Coupling effects, Transp. Porous Media 7 (1992) $63-82$.

[35] X. Olny, C. Boutin, Acoustic wave propagation in double porosity media, J. Acoust. Soc. Am. 113 (2003) 73-89.

[36] D.L. Sounas, R. Fleury, A. Alú, Unidirectional cloaking based on metasurfaces with balanced loss and gain, Phys. Rev. Appl. 4 (2015) 014005.

[37] D.R. Smith, S. Schultz, P. Markos, C.M. Soukoulis, Determination of effective permittivity and permeability of metamaterials from reflection and transmission coefficients, Phys. Rev. B: Condens. Matter 65 (2002) 195104.

[38] V. Fokin, M. Ambati, C. Sun, X. Zhang, Method for retrieving effective properties of locally resonant acoustic metamaterials, Phys. Rev. B: Condens. Matter 76 (2007) 144302.

[39] B.-I. Popa, S.A. Cummer, Design and characterization of broadband acoustic composite metamaterials, Phys. Rev. B: Condens. Matter 80 (2009) 174303.

[40] M. Yang, G. Ma, Y. Wu, Z. Yang, P. Sheng, Homogenization scheme for acoustic metamaterials, Phys. Rev. B: Condens. Matter 89 (2014) 064309.

[41] C. Chesnais, C. Boutin, S. Hans, Effects of the local resonance on the wave propagation in periodic frame structures: Generalized Newtonian mechanics, J. Acoust. Soc. Am. 132 (2012) 2873-2886.

[42] J. Soubestre, C. Boutin, Non-local dynamic behavior of linear fiber reinforced materials, Mech. Mater. 55 (2012) 16-32.

[43] R. Courant, D. Hilbert, Methods of Mathematical Physics, Interscience Publishers, Inc., 1953.

[44] B. Gambin, E. Kröner, Higher-order terms in the homogenized stress-strain relation of periodic elastic media, Phys. Status Solidi (B) 151 (1989) 513-519.

[45] C. Boutin, Sound propagation in rigid porous media: Non-local macroscopic effects versus pores scale regime, Transp. Porous Media 93 (2010) $309-329$.

[46] V. Romero-García, G. Theocharis, O. Richoux, A. Merkel, V. Tournat, V. Pagneux, Perfect and broadband acoustic absorption by critically coupled subwavelength resonators, Sci. Rep. 6 (2016) 19519.

[47] J.-P. Groby, R. Pommier, Y. Auregan, Use of slow sound to design perfect and broadband passive sound absorbing materials, J. Acoust. Soc. Am. 139 (2016) 1660-1671.

[48] J.-L. Auriault, Effective macroscopic description for heat conduction in periodic composites, Int. J. Heat Mass Transfer 26 (1983) $861-869$.

[49] F.J. Fahy, C. Schofield, A note on the interaction between a Helmholtz resonator and an acoustic mode of an enclosure, J. Sound Vib. 72 (1980)365-378.

[50] A. Cummings, The effects of a resonator array on the sound field in a cavity, J. Sound Vib. 154 (1992) 25-44.

[51] J.P.D. Hartog, Mechanical Vibrations, Dover Publications, 1985, Reprint of the McGraw-Hill 1956 edition.

[52] C. Zwikker, C.W. Kosten, Sound Absorbing Materials, Elsevier Publishing Company, Inc., 1949.

[53] A. Selamet, I. Lee, Helmholtz resonator with extended neck, J. Acoust. Soc. Am. 113 (2003) 1975-1985.

[54] A. Krynkin, O. Umnova, A.Y.B. Chong, S. Taherzadeh, K. Attenborough, Scattering by coupled resonating elements in air, J. Phys. D: Appl. Phys. 44 (2011) 125501.

[55] C. Ding, H. Chen, S. Zhai, S. Liu, X. Zhao, The anomalous manipulation of acoustic waves based on planar metasurface with split hollow sphere, J. Phys. D: Appl. Phys. 48 (2015) 045303.

[56] J.-P. Groby, W. Lauriks, T.E. Vigran, Total absorption peak by use of a rigid frame porous layer backed by a rigid multi-irregularities grating, J. Acoust. Soc. Am. 127 (2010) 2865-2874. 